Pierre Schneider

Univ. Artois, UR 4027, Centre de Recherche et d'Etudes Histoire et Sociétés (CREHS), F-62000

Arras,

France

Article disponible sur le portail Persée : https://www.persee.fr/doc/topoi 1161 -

94732006 num 1412153

\title{
La connaissance des mangroves tropicales dans l'Antiquité
}

Les mangroves tropicales, dont il faut aujourd'hui déplorer l'inexorable destruction, ne pouvaient échapper à I'inlassable curiosité des Anciens (particulièrement celle des Grecs) qui naviguaient le long des rivages de I'océan Indien. Quelques débris de leurs connaissances, quelques traces de leur étonnement, ont survécu. Parmi les Modernes, H. Bretzl et S. Amigues ont su, par leurs vigoureux commentaires, honorer la valeur des témoignages antiques. La synthèse que nous proposons ici était inconcevable sans leurs enseignements.

\section{I) Les informateurs}

\section{1) L'époque d'Alexandre}

Il est possible que, dès le VI ${ }^{\mathrm{e}}$ siècle av. J.-C., des Grecs aient pu apercevoir des mangroves ${ }^{1}$. Mais les témoignages font défaut. Mieux vaut alors croire que la campagne orientale d'Alexandre marque le point de départ des connaissances antiques. Tout, en effet, laisse penser, en l'état actuel de la documentation, que les mangroves ne furent vraiment découvertes et approchées que dans la phase finale de l'expédition d'Alexandre, et que les descriptions n'en furent diffusées qu'après celle-ci. L'apport des Gréco-Macédoniens a, dans sa majeure partie, été préservé par Théophraste, qui présente une sorte de bilan des découvertes des compagnons d'Alexandre ( $\hat{0} \mathscr{D}$

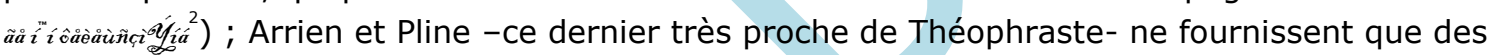
compléments d'information.

Qui furent les informateurs? Comme souvent, on les identifie mal et l'on ne parvient à en nommer quelques-uns qu'au prix de conjectures plus ou moins heureuses. Pour ce faire, nous adopterons un découpage en trois ensembles géographiques : seront successivement examinés le secteur de la mer d'Oman et de la rive iranienne du golfe Persique (mer Érythrée), puis celui de Bahrein (Tylos) et enfin celui de la mer Rouge (golfe Arabique) ${ }^{3}$. Au reste, cette division ne nuit pas à l'enquête. En effet, les observateurs ne furent généralement pas les mêmes d'une région à l'autre.

1.1) Les mangroves de la mer d'Oman et de la rive orientale du golfe Persique : Néarque ; Androsthène ; Onésicrite; Aristobule.

Durant l'été 325, Alexandre divisa son armée en deux corps en vue du retour vers Babylone: I'un, commandé par le roi, devait partir en septembre et effectuer le trajet par la voie terrestre en longeant le littoral ; I'autre, un groupe naval dirigé par Néarque, devait partir au même moment et rallier Babylone par la mer ; ce dernier fut retardé d'un mois en raison des vents contraires de la mousson d'été finissante. L'itinéraire maritime, dont le delta de l'Indus constituait le point de départ, passait par la Gédrosie, la Carmanie, le détroit d'Ormuz et la côte iranienne du golfe Persique. Les deux groupes firent jonction en décembre 325, à Alexandrie de Carmanie, près d'Ormuz. Néarque parvint à Suse vers la mi-février $324^{4}$. Les hommes des deux groupes

Nous remercions vivement $M^{\text {lle }}$ Suzanne Amigues d'avoir accepté de lire ce travail en cours d'élaboration et de nous avoir fait bénéficier de ses précieuses remarques.

1 Vers 520, Scylax de Caryanda effectua une navigation de l'Indus à l'Égypte pour le compte de Darius le Grand. Par ailleurs, des Grecs étaient dès le VI ${ }^{\mathrm{e}}$ siècle établis en Égypte (voir infra, p. )

2 Théophraste, H. P., 4, 7, 8.

3 Figurent entre parenthèses les noms ordinairement en usage dans la géographie antique. Noter cependant que le nom «mer Érythrée » peut être appliqué extensivement aux golfes Arabique et Persique.

4W. Capelle, R.E., s.v. Nearchos, 2142 (I'auteur se réfère à H. Bretzl). Voir aussi P. Pédech, La découverte de I'Inde, p. 188 (en juillet 325 Alexandre se trouve à Pattala) ; p. 195 (arrivée à l'embouchure de I'Euphrate à la mi-mai 324). 
rencontrèrent sur leur trajet des zones de mangroves et plusieurs ne manquèrent pas de consigner leurs observations ${ }^{5}$.

Cependant, les membres de l'expédition navale contribuèrent plus que les autres à faire connaître aux Grecs les mangroves de ce secteur. On le perçoit à la richesse des informations de Théophraste, lequel, à n'en pas douter, s'appuie principalement sur des témoignages de

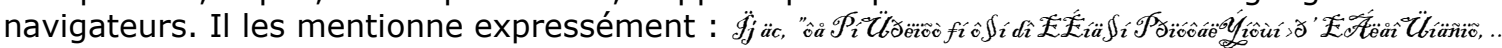
¿áóéí . Par ailleurs, certains détails, dans son exposé, dévoilent un point de vue de marin. Ainsi est-il fait mention d'amarres, que les matelots pouvaient accrocher au sommet de certains arbres à marée haute et à leurs racines à marée basse, ou d'îles soumises aux marées ${ }^{7}$. Qu'il s'agisse d'îles véritables, situées entre les bouches de l'Indus et la Perse ${ }^{8}$, ou simplement de bancs de vase découverts à marée basse ${ }^{9}$, les informations proviennent de reconnaissances navales. Au demeurant, il n'est guère surprenant que de telles observations proviennent de navigateurs. Reste à tenter de les identifier.

Théophraste, notre source principale, ne nomme personne. Pline n'apporte aucune précision supplémentaire : qui navigare in Indos Alexandri milites...tradidere ${ }^{10}$. Par chance, grâce à Arrien, nous connaissons la liste de ceux qui participèrent comme chefs de trière à l'expédition commandée par Néarque ${ }^{11}$. C'est donc parmi ceux-ci, à défaut d'autres qui nous soient connus ${ }^{12}$, qu'il faut chercher les sources d'information.

Le nom de Néarque vient immédiatement à l'esprit. Selon certains, il est même la source unique de Théophraste pour ce secteur maritime. F. Jacoby a ainsi rangé les quatre paragraphes de Théophraste qui nous intéressent ${ }^{13}$ au nombre des fragments de Néarque ${ }^{14}$. Mais trois objections rendent cette thèse fragile. Tout d'abord, Théophraste (et Pline) se réfèrent ouvertement à une

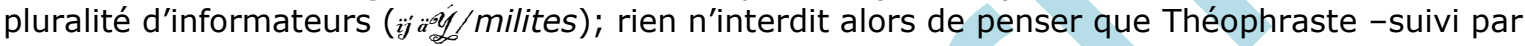
Pline- utilisa diverses relations composées par des membres de l'expédition navale curieux d'histoire naturelle (il n'en manquait pas). Ensuite : que Théophraste fasse mention, en début d'exposé, du voyage de retour des soldats d'Alexandre ne suffit pas à placer sous l'autorité de Néarque tout ce qui suit. Enfin, on a noté que si ce passage de Théophraste était bien un fragment de Néarque, il ne ressemblerait guère aux autres, d'attribution certaine. Ces derniers n'ont pas la précision du texte de Théophraste ${ }^{15}$. En effet, la seule mention de la mangrove qui puisse être

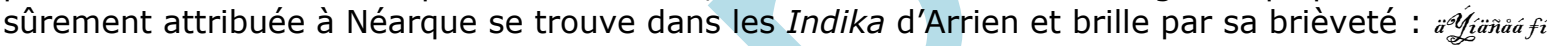

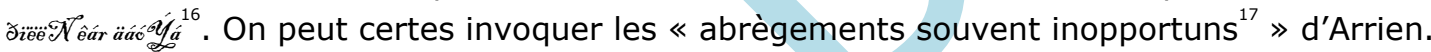

Néanmoins, si Néarque avait soigné son exposé sur les mangroves, comment comprendre qu'Arrien n'en retienne à peu près rien, et surtout rien de ce qui les rendait spectaculaires ? Concluons en prenant appui sur le texte de Théophraste : parmi les membres de l'expédition navale, Néarque

\footnotetext{
5Théophraste, H.P., 4, 7, 3-6 ; Pline, 12, 37 (cf. Théophraste, H.P. 4, 7, 5-6 [Pline cite Théophraste dans la liste des auteurs étrangers qu'il a consultés) ; 13, 140-141 (cf. Théophraste, H.P., 4, 7, 3-5); Arrien, Anab., 6, 22, 6-7.

6 Cf. L. Pearson, Lost Histories, p. 142, n. 104.

7 Théophraste, H. P., 4, 7, 4 (comparer avec Pline, 13, 141).

8 Les navigateurs de l'expédition mouillèrent dans plusieurs îles (Arrien, Ind., 22, $2 ; 22,7 ; 31,5$; 37, 7 ; 38,2 ). Selon H. Bretzl, B.F., p. 31-34, les îles que mentionne Théophraste (loc. cit. supra, n. 7 ) se trouvent dans le delta de I'Indus mais le texte n'invite pas à une interprétation aussi restrictive.

9 Voir Diodore de Sicile, $17,106,6$.

10 La formulation de Pline est surprenante car, lorsqu'il se rendit "chez les Indiens", Alexandre n'emprunta pas un itinéraire maritime. Soit Pline se méprend, soit il estime que, naviguant sur l'océan Indien, éponyme des peuples qui en occupaient le littoral, les marins d'Alexandre effectuaient -au moins en partie- un voyage qui les menait "chez les Indiens". Voir H. Bretzl, B.F., p. 86-87 et la note d'A. Ernout (Pline, Histoire naturelle, livre 13, Paris, 1956, p. 115, n. du § 140). Sur une telle conception de l'océan Indien chez Pline, voir Id., 6, 33.

11 Arrien, Ind., 18, 1-10. Pline nomme dans ses sources des livres 12 et 13 des membres de l'expédition d'Alexandre (Néarque, Anaximène, Onésicrite, Charès de Mytilène). Cf. S. Amigues, Recherches, t. 1, p. XXV.

12 Par exemple, nous ignorons quels hommes firent la reconnaissance des îles du delta de l'Indus (Arrien, Anab., 6, 19)

13 Théophraste, H. P., 4, 7, 3-6.

14 F.Gr.H. 133, F34. Cf. également H. Bretzl, B.F., p. 23-24 ; p. 37-64 ; p. 181 ; P. Pédech, Historiens, p. 203.

15 L. Pearson, Lost Histories, p. 142, n. 104 ; H. Strasburger, Alexanders Zug, p. 464 ; S. Amigues, Recherches, t. 1, p. XXIV-XXV.

16 Arrien, Ind., 22, 7 (120 stades environ avant l'embouchure du fleuve Arabis).

17 P. Pédech, Historiens, p. 203, n. 18.
} 
rapporta certainement des renseignements sur la mangrove, mais il ne dut pas être le seul. Qui étaient les autres?

W. Capelle et d'autres préféraient à Néarque Androsthène de Thasos, dont nous reparlerons plus loin, à propos de Tylos ${ }^{18}$. En effet, Androsthène avait participé à la navigation de I'Indus à I'Euphrate comme chef de trière et avait rédigé un Paraplous tês Indikês. On sait également que Théophraste connaissait son ouvrage. S. Amigues souligne ses qualités d'observateur et de naturaliste. W. Capelle ajoute que, les paragraphes 4, 7, 7-8 de l'Histoire des Plantes [mangroves de Tylos], indiscutablement pris à Androsthène, faisant immédiatement suite aux quatre qui nous préoccupent ici $(4,7,3-6)$, il n'est pas absurde de penser que ces derniers soient aussi empruntés à la même source. Il est donc probable, en raison de ces quatre arguments, qu'Androsthène a renseigné Théophraste au sujet des mangroves de la mer d'Oman. On remarque toutefois quelques discordances entre les paragraphes I'H.P. consacrés à Tylos et ceux consacrés à la mer d'Oman. Par exemple, dans le paragraphe 7, la végétation de mangrove est faiblement mise en relation avec son biotope marin (une seule allusion à la submersion par le flux apparaît), ce qui contraste avec les paragraphes 3-6 (mer d'Oman), où est décrite avec détails l'association intime des végétaux et de la mer. Certains renseignements de Théophraste pourraient donc provenir d'autres informateurs.

Onésicrite fait figure de candidat sérieux, malgré l'opprobre dont il est généralement couvert. Il a participé à la navigation de retour vers l'Euphrate, a rédigé un ouvrage consacré à Alexandre, parsemé de données tantôt pertinentes, tantôt fantaisistes. Malgré tout, on lui reconnaît des compétences d'histoire naturelle, particulièrement en botanique ${ }^{19}$. Aussi est-il concevable que certaines lignes de Théophraste soient empruntées à Onésicrite. Dire lesquelles est hasardeux : il pourrait s'agir aussi bien de réflexions théoriques que d'exagérations, car l'on trouve des exemples des deux sortes. Ainsi, dans tel passage, on tente d'expliquer rationellement comment les espèces de mangrove peuvent survivre en milieu salé ; ailleurs, on lit certains arbres sont grands comme les plus hauts platanes et les plus hauts peupliers, et que seuls les plus grands individus échappent, à marée haute, à la submersion totale, ce qui est invraisemblable ${ }^{20}$. Tout ceci pourrait émaner d'Onésicrite, mais aucune preuve ne s'impose. À la lumière du texte de Théophraste, on peut toutefois admettre avec sûreté que la plupart des renseignements sur les mangroves de la mer d'Oman proviennent de divers navigateurs, dont au moins trois peuvent être nommés sans heurter la vraisemblance : Androsthène, Onésicrite, Néarque.

Il est possible, sans compter ceux dont les noms ont disparu, de citer d'autres sources potentielles d'information : Ptolémée, Clitarque, Archias de Pella, Anaximène, Charès de Mytilène, Orthagoras, mais leur présence est indécelable dans les documents qui ont survécu. Reste cependant le cas d'Aristobule, qui a fait partie du corps expéditionnaire terrestre. Ce compagnon d'Alexandre a décrit les mangroves de Gédrosie, comme l'atteste le texte d'Arrien ${ }^{21}$. Mais, à la différence des navigateurs auxquels se réfère Théophraste, il a décrit cette végétation (qu'il a observée ${ }^{22}$ ) avec le point de vue d'un voyageur pédestre. En effet, dans la notice d'Arrien, la mangrove s'insère dans un panorama botanique consacré à la végétation du désert de Gédrosie :

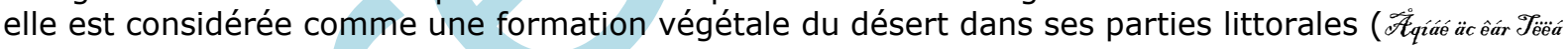

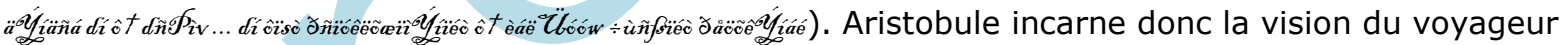
qui découvre ce paysage littoral en arrivant de l'intérieur des terres, à l'opposé des hommes qui informent Théophraste. Ceci n'empêche pas des convergences entre Aristobule (bon observateur et

\footnotetext{
18 W. Capelle, R.E., s.v. Nearchos, 2148-2149, suivi par S. Amigues, Recherches, t. 1, XXVIII-XXIX ; Regenbogen, R.E., Suppl. 7, s.v. Theophrastos, 1462.

19 T.S. Brown, Onesicritus, p. 70-104 ; P. Pédech, Historiens, p. 146-150. Il est probable que Théophraste s'est servi, pour ses recherches, de la relation d'Onésicrite (cf. H. Strasburger, Alexanders Zug, p. $464 ; \mathrm{S}$. Amigues, Recherches, t. 1, p. XXVI et n. 55, au sujet du banyan).

20 S. Amigues, Recherches, t. 2, p. 257. Cf. également infra, p. .

21 Arrien, Anab., 6, 22, 6-7 (comparer avec Théophraste, H. P., 4, 7, 4). Il est généralement admis que I'ouvrage d'Aristobule a dû paraître trop tard pour que Théophraste pût en tenir compte dans les derniers remaniements de son œuvre (H. Strasburger, Alexanders Zug, p. 460-461 ; L. Pearson, Lost Histories, p. 152 ; S. Amigues, Recherches, t. 1, p. XXVII-XXVIII).

22 Ce point est discuté. L. Pearson, Lost Histories, p. 177 et n. 146 (à la suite de H. Bretzl, B.F., p. 78-79 et suivi par P. Pédech, Historiens, p. 400) juge qu'Aristobule a décrit ce qu'il a vu (contra cf. F. Jacoby, F.Gr.H. 139, note du F49 : Jacoby estime qu'Aristobule fait un emprunt à Néarque ; cf. aussi $\mathrm{H}$. Strasburger, Alexanders Zug, p. 462, n. 2). P. Pédech, op. cit., p. 400 rappelle que d'après Arrien, Anab., 6, 26, 5, l'expédition parcourut le littoral gédrosien sept jours durant (voir également Arrien, Anab., 6, 23, 1-3). Il subsiste aujourd'hui trois zones de mangrove dans le Balouchistan oriental (Miani Hor ; Khalmot Hor ; Gawater Bay : cf. WMA, p. 63). Pour H. Bretzl, op. cit., p. 79, Aristobule aurait pu faire ses observations dans la première des trois, qui est aussi la plus étendue.
} 
excellent naturaliste ${ }^{23}$ ) et les auteurs qu'utilise Théophraste : sans doute les uns et les autres ontils fait des observations semblables, à moins qu'ils n'aient échangé des informations ; il se peut également qu'Aristobule, qui a écrit sa relation tardivement, ait fait des emprunts à ceux de ses compagnons qui avaient publié leur livre avant lui.

\section{2) Les mangroves de Bahrein (Tylos): Androsthène ; Hiéron ; Archias.}

Peu de temps après le retour de la flotte de Néarque, Alexandre voulut réaliser un projet de circumnavigation de I'Arabie. Trois expéditions furent lancées dès l'automne 324, qui devaient longer la rive occidentale du golfe Persique, totalement inconnue des Grecs ${ }^{24}$. Elles furent conduites par Archias de Pella, Androsthène de Thasos ${ }^{25}$, Hiéron de Soloi ${ }^{26}$. Tous rapportèrent leurs renseignements à Alexandre et nous sommes assurés par Arrien que les deux premiers passèrent à Tylos. De sérieux indices montrent que les mangroves de Tylos (aucun témoignage ne fait mention d'autres mangroves sur la rive occidentale du golfe Persique) furent décrites par Androsthène et que Théophraste exploita cette relation ${ }^{27}$. A-t-il utilisé d'autres sources, ce qui reviendrait à dire qu'Androsthène ne fut pas le seul à transmettre des observations sur les mangroves de Bahrein ? De fait Théophraste utilise le verbe öácé. Admettons que ce pluriel désigne une pluralité d'auteurs. S'est-il servi de Hiéron de Soloi ? Il est impossible de se prononcer sur ce point. En revanche, des emprunts à Archias sont douteux. En effet, cet explorateur ne dut pas apercevoir de mangrove, puisqu'il rapporta à Alexandre que l'île, propre à porter des cultures, était dépourvue de forêts ${ }^{28}$ : soit il n'aborda pas l'île du même côté qu'Androsthène, soit, hypothèse plus surprenante, il ne considérait pas les mangroves comme des formations forestières. Quoiqu'il en soit de ces suppositions, son utilisation par Théophraste doit donc être écartée. On considérera donc, à la suite de H. Bretzl, W. Capelle et d'autres, qu'Androsthène fut la principale, sinon la seule, source $d^{\prime}$ information pour les forêts littorales de Bahrein ${ }^{29}$. Il ne découvrait d'ailleurs rien de nouveau puisqu'il avait eu tout loisir d'apercevoir ce genre de formation végétale en accompagnant Néarque.

\section{3) Les mangroves de la mer Rouge (golfe Arabique).}

Au moment où les hommes d'Alexandre se lançaient à la découverte de la rive occidentale du golfe Persique et de Bahrein, d'autres étaient chargés de faire le tour de la péninsule arabique depuis l'Égypte. D'après W.W. Tarn, à l'époque où Hiéron entamait son périple, une expédition d'exploration quittait Heroônpolis, avec la mission de contourner la péninsule arabique ${ }^{30}$. Un certain Anaxicrate, I'un des compagnons d'Alexandre, la conduisit, jusqu'au Bal el-Mandeb où il lui fallut faire demi-tour. Le voyage se déroula durant l'année $324^{31}$. Cette expédition permit-elle aux voyageurs de découvrir des zones de mangrove dans le golfe de Suez ${ }^{32}$ ?

S. Amigues juge recevable cette hypothèse qu'elle fonde sur le texte de Théophraste («Dans le golfe des Héros, où descendent ceux qui partent d'Égypte... ») car, d'après elle, il faut

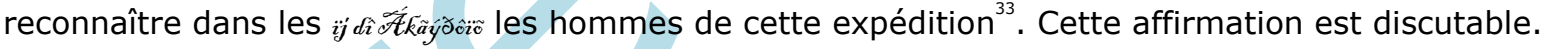
On observera en effet que Théophraste utilise un verbe au présent (êấćâấf̧izióóéi). Par comparaison, Arrien faisant allusion, selon toute vraisemblance, à l'expédition d'Anaxicrate se sert de l'aoriste ${ }^{34}$. Si le présent de Théophraste correspond à un fait habituel, ne s'agirait-il pas plutôt de simples voyageurs anonymes ? De surcroît, le propos de Théophraste n'incite guère à reconnaître la

\footnotetext{
23 S Amigues, Recherches, t. 1, p. XXVI ; L. Pearson, Lost Histories, p.177. Sur la qualité de ses observations, voir infra, $\mathrm{p}$.

24 J.-F. Salles, Découvertes, p. 184.

25 On admet à la suite de $\mathrm{H}$. Bretzl qu'Androsthène fit halte à Tylos de décembre 324 à janvier 323. S. Amigues, Recherches, t. 2, p. 259, propose une datation légèrement plus haute (octobre-novembre 324).

26 Arrien, Anab., 7, 20, 6-8; Strabon, 16, 3, 2.

27 Théophraste, H.P., 4, 7, 7-8 (et comparer avec Pline, 12, 38-40). Voir supra, p. et n. 18.

28 Arrien, Anab., 7, 20, 6. Selon K. Karttunen, India, p. 45, il semble qu'Archias n'ait rien écrit.

29 On ignore si la description de Tylos faisait partie du Paraplous tês Indikês.

30 W.W. Tarn, Ptolemy II, p. 13-14. Cependant, certains éléments de l'enquête de W.W. Tarn sont remis en cause par P. Högemann, Arabien, p. 83, n. 9

31 P. Högemann, Arabien, p. 83 (départ avant octobre 324). Pour un examen de la chronologie à l'aide des renseignements botaniques, voir S. Amigues, Études, p. 57-62.

32 Théophraste, H.P., 4, 7, 2.

33 S. Amigues, Recherches, t. 1, p. 252.

34 Arrien, Ind., 43, 7.
} 


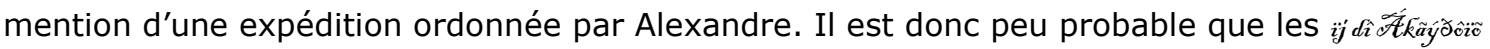
soient les membres de l'expédition d'Anaxicrate. Ce n'est pas tout : ces « hommes qui partent d'Égypte » ne sont pas présentés comme source d'information par l'intermédiaire d'un verbe comme đä́ó (à la différence des compagnons d'Alexandre pour le secteur de la mer d'Oman): ce groupe nominal apporte simplement au lecteur une précision pour mieux situer le « golfe des Héros ». Il nous paraît donc douteux qu'Anaxicrate ait informé Théophraste. De fait, il n'était pas nécessaire d'avoir parcouru la côte arabique jusqu'au Bab el- Mandeb pour fournir des renseignements sur la végétation du golfe de Suez ; bien des Grecs, installés en Égypte avant I'époque d'Alexandre, avaient pu apporter des informations. Il n'est en effet pas invraisemblable qu'une mangrove située dans cette zone ne fût connue depuis quelque temps, surtout si elle était exploitée pour fournir des substances médicinales ${ }^{35}$. Ce passage de Théophraste, loin de montrer que l'expédition dirigée par Anaxicrate ait été à l'origine d'observations, nous autorise seulement à supposer que les mangroves du golfe de Suez étaient connues avant l'expédition d'Alexandre.

Théophraste signale par ailleurs une zone de mangrove sur la rive occidentale de la mer Rouge. Il mentionne, sans citer d'informateur, des «lauriers » et des « oliviers », qui poussent « au-dessus de Coptos », «dans I'Arabie qui fait partie de la mer dite Érythrée»36. Que peut

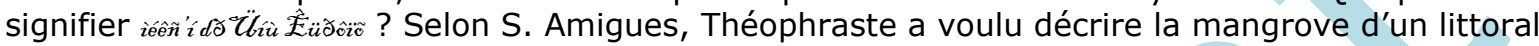
de la mer Rouge qu'il situait au sud de la latitude de Coptos : " "au-dessus de Coptos" ne signifie pas exactement "en amont de C.", mais "au sud par rapport à la latitude de C." $\gg^{37}$. Il faudrait alors admettre que Théophraste se réfère ici à une perception abstraite de l'espace, celle de la géographie mathématique et astronomique, celle qui prend pour repères cartographiques les parallèles, les « climats » et la mesure des jours solsticiaux.

Une autre interprétation paraît recevable, en relation avec une autre perception de I'espace, celle de l'itinéraire: «au-dessus de Coptos » signifie dans ce cas « après Coptos [i.e. pour celui qui parcourt un chemin terrestre] ». Le texte de Pline ne s'oppose pas à ce choix : At in oriente (i.e. par opposition à la mer occidentale située en dehors des colonnes d'Hercule) mirum est statim a Copto per solitudines nihil gigni praeter spinam. Le texte de Théophraste prend alors un sens différent : celui qui chemine depuis Coptos vers la mer ne trouve rapidement plus d'arbres le long de son chemin (excepté un arbre qualifié d' « assoiffé »); en revanche, il en voit au bout de son itinéraire, qui poussent dans l'eau salée. La mangrove que décrit Théophraste ne serait pas celle d'un certain littoral situé au sud de la latitude de Coptos, mais celle que le voyageur rencontre au bord de la mer Rouge, quelque part au terme d'une piste partant de $\operatorname{Coptos}^{38}$. On sait que de telles pistes étaient utilisées dès la XI $\mathrm{I}^{\mathrm{e}}$ dynastie ${ }^{39}$. On peut par conséquent concevoir que des Grecs aient entendu parler très tôt de cette mangrove (d'après Hérodote, les Grecs commencèrent à obtenir des renseignements sur l'Égypte à partir du règne de Psammétique ${ }^{40}$ ), voire qu'ils l'aient observée eux-mêmes. Seraient-ce les informateurs de Théophraste?

\section{2) Après Alexandre}

L'époque d'Alexandre, véritable commencement des observations de la mangrove, en est aussi l'apogée. Les témoignages et les apports nouveaux se raréfient au cours de l'époque hellénistique. Dans l'état actuel de la documentation, les quelques observations relativement originales se limitent au secteur de la mer Rouge. En effet, aucune donnée nouvelle n'est connue pour le secteur de la mer d'Oman : Mégasthène n'a visiblement pas fait autre chose que répéter et simplifier ce que les compagnons d'Alexandre avaient écrit ${ }^{41}$. Dans la région du golfe Persique, la présence épisodique des Séleucides ${ }^{42} n^{\prime}$ a sans doute pas autorisé de nouvelles observations ${ }^{43}$. En revanche, l'intérêt des premiers Lagides pour la mer Rouge a permis d'accroître, médiocrement il est vrai ${ }^{44}$, la connaissance des mangroves. On en trouve des traces chez Agatharchide de Cnide,

\footnotetext{
35 Cf. infra, p. .

36 Théophraste, H. P., 4, 7, 1 (comparer avec Pline, 13, 139).

37 S. Amigues, Recherches, t. 2, p. 247-248.

38 H. Bretzl, B.F., p. 105, mentionne une mangrove située près de Qosseir $\left(26^{\circ} 5^{\prime} \mathrm{N}\right)$, au point extrême du Wadi Hammamat. Sur cette route très ancienne, voir J.-P. Brun, Hodos Myshormitikè, p. 397.

39 J. Desanges, Recherches, p. 239 ; M. Reddé et J.-Cl. Golvin, Du Nil à la mer Rouge, p. 5-64.

40 Hérodote, 2, 154.

41 Antigonos, Hist. mirab., 132 (= F. Gr. H. 715, F25).

42 J.-F. Salles, Découvertes, p. 89 ; Idem, Hellénisme, p. 223-232 ; R. Boucharlat et M. Mouton, Importations, p. 275-289.

43 Au sujet des renseignements qu'apporte Ératosthène (Strabon, 16, 3, 6), cf. infra, p. .

44 Cf. infra, p. .
} 
qui, à la fin du II ${ }^{\mathrm{e}}$ siècle av. J.-C., a bénéficié des renseignements collectés par des hommes au service de Ptolémée II et Ptolémée III (quelques-uns sont connus : Straton, Démétrios, Conon, Antiphilos, Simmias ${ }^{45}$ ).

Ainsi, Agatharchide signale des bois denses d'oliviers endémiques ( différents de ceux de chez nous ») dans des îles du nord de la mer Rouge. Seul le nom «olivier », déjà présent chez Théophraste, nous incite à supposer qu'il s'agit d'un arbre de mangrove, car Agatharchide n'indique pas leur relation intime avec le milieu marin ${ }^{46}$. Progressant vers le sud, il décrit de nouveau des oliviers dans la région des Ichtyophages et nous offre enfin quelques détails originaux sur cette végétation : feuillage dense, fruit semblable à la châtaigne ; les Ichtyophages utilisent ces arbres comme abri, ajoute-t-ilit. Agatharchide évoque enfin des oliviers de la région du Bab el-Mandeb. Dans ce passage, l'auteur n'apporte, du point de vue botanique, aucun élément nouveau : les données qu'il fournit coïncident avec les observations faites à l'époque d'Alexandre. En effet, on savait déjà que ces arbres étaient immergés, voire submergés, à marée haute. Pour être cependant exact, il faut reconnaître qu'avec Agatharchide l'existence de la marée en mer Rouge est pour la première fois mis en valeur.

On conclura donc que les officiers et chasseurs au service de Ptolémée II et de Ptolémée III ont su noter, au fur et à mesure de leurs progrès vers le Bab el-Mandeb, l'existence de zones littorales boisées et les décrire sommairement. Mais, en dehors du traité d'Agatharchide, on ne trouve presque rien. Les données d'Artémidore, transmises par Strabon, sont sommaires et dépendent pour partie d'Agatharchide ${ }^{48}$. On connaît aussi le traité Sur la mer Érythrée d'un certain Pythagoras, dont la période d'activité pourrait se situer vers 220 av. J.-C. Décrivit-il les mangroves de la mer Rouge? Nous l'ignorons. Seule en tout cas a subsisté, grâce à Athénée, une phrase dans laquelle l'auteur parle des « oliviers marins » qu'utilisent les Troglogytes pour confectionner

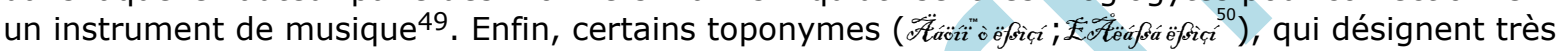
vraisemblablement des zones de mouillage à proximité de mangroves, nous ont été transmis.

Dans l'état actuel de la documentation, très rares sont les données postérieures à l'époque hellénistique. La mer Rouge et l'océan Indien ont été traversés par de nombreux marchands sans que, semble-t-il, les connaissances antiques aient progressé. Peut-être ces hommes n'y prêtaientils pas attention à cet élément du paysage. Ainsi l'auteur du Périple de la mer Érythrée connaît un

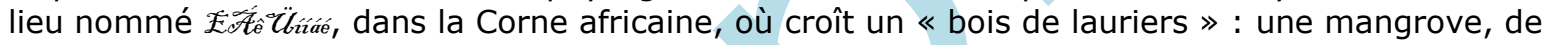
toute évidence ${ }^{51}$. Mais ce commerçant, que l'on voit parfois s'intéresser à l'histoire naturelle, ne donne ici aucun renseignement. Pline ajoute très peu aux connaissances hellénistiques : il rapporte des informations en provenance de Taprobane ${ }^{52}$ mais ses renseignements sont banals, sommaires et indirects. Tout ce que Pline sait a été transmis par des ambassadeurs qu'avait envoyés à Rome le souverain de l'île, Rachias. Aux I ${ }^{\mathrm{er}}$-II ${ }^{\mathrm{e}}$ siècles, Plutarque sait très approximativement ce qu'est la mangrove ${ }^{53}$. Sans doute ne s'intéressait-on plus beaucoup à ces paysages.

\section{II ) La valeur des connaissances antiques}

\section{1) L'apport supérieur de l'expédition d'Alexandre}

Comme on vient de le voir, la très grande majorité des observations de la mangrove remonte à l'expédition d'Alexandre (à partir de 325) et à l'époque des royaumes hellénistiques (en particulier celle des explorations lagides en mer Rouge). C'est un constat étonnant, si I'on songe que les progrès de la navigation commerciale (à partir du $\mathrm{I}^{\mathrm{er}}$ siècle av. J.-C.) ${ }^{54}$, mirent à portée des

\footnotetext{
45 Bibliographie abondante sur ce sujet. Cf. par ex. J. Desanges, Recherches, 274-275.

46 Photius, 456b (= Agatharchide, Érythr., 81) ; 457b (= Agatharchide, Érythr., 91) ; Diodore de Sicile, 3, 39, 2.

47 Photius, 450b (= Agatharchide, Érythr., 43) ; Diodore de Sicile, 3, 19, 3 ; comparer avec Strabon, 16, 4, 13 in fine.

48 Strabon, $16,4,14 ; 16,4,18$.

49 Athénée, 4, 82, 184a. Nous ne pouvons que regretter la perte des monographies de Juba, qui utilisait principalement des sources d'époque hellénistique. Ce qui nous en est resté (Pline, 13, 142 et peut-être 12, 77) ne nous apprend rien de véritablement nouveau.

50 Strabon, 16, 4, 8 ; 16, 4, 14 ; Stéphane de Byzance, s.v Elaia.

51L. Casson, The Periplus, p. 129.

52 Pline, 6, 87 ; Solin, 53, 21. Cf. D. P. M. Weerakkody, Taprobanê, p. 68.

53 Cf. infra, p. .

54 En relation avec la découverte (à une date discutée) des cycles de la mousson.
} 
navigateurs méditerranéens des régions riches en mangroves : l'Afrique orientale, le golfe du Bengale, Ceylan. C'étaient, de surcroît, des mangroves plus spectaculaires et plus variées que celles de la mer Rouge, du golfe Persique et de la côte irano-pakistanaise, naturellement pauvres en raison des difficiles conditions naturelles (aridité, salinité), sinon appauvries par I'homme.

Cependant, du point de vue qualitatif, les observations des membres de l'expédition orientale d'Alexandre ${ }^{55}$ l'emportent sur celles de l'âge hellénistique (principalement celles de l'époque ptolémaïque). Les premières révèlent un authentique souci du détail et de la précision descriptive. Les observateurs qui ont accompagné Alexandre paraissent être des hommes instruits et curieux : ils se sont vraisemblablement interrogés sur les effets de l'eau salée, s'appuyant peutêtre sur des notions de physique ancienne ${ }^{56}$; ils se sont sans doute livrés à des manipulations, car, à lire Théophraste, on imagine aisément tel membre de l'expédition navale ouvrant le fruit d'un Avicennia pour en observer l'intérieur, tel autre creusant la vase pour examiner l'enracinement d'un arbre ${ }^{57}$. En revanche, dans les témoignages de l'époque hellénistique, les observations sont plus générales et plus sommaires, les précisions botaniques (fleur, fruit, feuille) rares, le volume d'information réduit, la démarche scientifique absente. Aussi les descriptions de l'époque ptolémaïque (mangroves de la mer Rouge) ne valent-elles pas en qualité celles de l'époque d'Alexandre (mangroves de la mer d'Oman et du golfe Persique).

Comment rendre raisonnablement compte de ces différences qualitatives ? La supériorité des informations que transmirent les compagnons d'Alexandre n'est pas fortuite. Naturellement, leur qualité d'observateur compte pour beaucoup : ces voyageurs ont personnellement vu la mangrove et ont consigné eux-mêmes leurs observations. De I'« autopsie » à l'écrit, il n'y eut pas de détours inutiles. Mais cette « autopsie » fut soutenue par la valeur personnelle et la culture de ces hommes. On discerne chez eux une insatiable curiosité, relayée et soutenue par l'intérêt que le roi lui-même portait aux choses de la nature. Le désir agonistique de briller plus que les autres en fournissant des observations inédites l'attisait peut-être davantage. Il y eut donc, durant ces quelques mois des années 325-324, une miraculeuse conjonction de circonstances favorables.

Cette situation exceptionnelle ne se reproduira plus, pas même lors des explorations lagides en mer Rouge. Celles-ci en effet ne ressemblaient guère aux voyages de l'époque d'Alexandre. Le contexte, les objectifs, les hommes avaient changé. On doit notamment supposer que les informateurs lagides, dont nous ignorons certes les compétences et les personnalités, avaient une moindre curiosité scientifique que les gens de l'expédition d'Alexandre. Il faut bien, en effet, constater que, dans les témoignages de l'époque ptolémaïque, les arbres sont plus nommés que décrits, à l'inverse des compagnons d'Alexandre qui décrivent avec précision des arbres qu'ils ne nomment pas. L'énumération des mangroves qui parsèment la rive africaine de la mer Rouge (voir le texte de Strabon ${ }^{58}$ ) donne l'impression que celles-ci représentaient plus des repères de navigation ou des lieux de mouillage qu'elles ne suscitaient d'intérêt scientifique. On peut trouver ici une explication, au moins partielle, de la relative médiocrité des descriptions de l'époque lagide.

\section{2) La valeur des recherches de Théophraste}

On ne saurait trop louer l'exposé que Théophraste a consacré à la mangrove. Sans lui, les connaissances fondamentales qui ont été collectées jusqu'à l'époque d'Alexandre auraient certainement été perdues. On constate en effet que des savants qui ont compulsé les mêmes sources que lui (Diodore, Strabon ou Arrien) ne retiennent à peu près rien de ce paysage. De plus, il est parvenu à rassembler des notices variées et certainement dispersées pour composer un exposé synthétique - tâche qui révèle une intelligence hors pair, d'autant plus qu'il s'aventurait dans un domaine botanique aussi nouveau qu'embarrassant -. Enfin, alors que personnellement il n'a observé aucun des arbres dont il parlait, il a su poser les questions primordiales et proposer soit des conclusions, soit des hypothèses. Quelles étaient précisément ces questions?

En premier lieu, quel statut accorder à cette formation végétale ? La réponse ne va pas de soi, s'agissant d'arbres que rien ne distingue de leurs congénères terrestres, sauf qu'ils sont

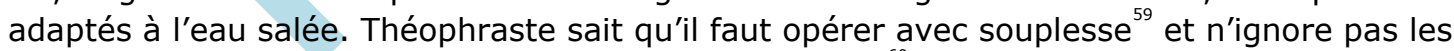
incertitudes que soulèvent les classifications botaniques ${ }^{60}$. Accordant la primauté au critère du

55 Ce qui ne signifie pas pour autant, comme on le verra plus loin (infra, p. ), qu'elles soient faciles à utiliser du point de vue botanique.

56 Cf. infra, p. .

57 Voir Théophraste, H. P., 4, 7, 5-6.

58 Strabon, $16,4,4 ; 7 ; 8 ; 13$ (si l'on suppose que le « bois » [alsos] d'Eumène désigne une mangrove) ; $14 ; 18$.

59 Cf. Théophraste, H. P., 1, 3, 5 : «Il y a lieu de ne pas s'en tenir trop rigoureusement à la définition et de considérer nos distinctions comme un simple schéma » (trad. S. Amigues).

60 Cf. S. Amigues, Études, p. 5. 
biotope (« chaque plante recherche une situation et un climat à sa convenance ${ }^{61} \gg$ ), il sépare les plantes terrestres des plantes aquatiques et range les arbres de mangrove dans la catégorie des plantes aquatiques d'eau salée ${ }^{62}$. Au sein de cette catégorie, Théophraste établit une distinction à la fois botanique et géographique : d'une part les plantes marines de Méditerranée et de l'Atlantique, petites et submergées ; d'autre part celles qui croissent dans la mer Extérieure (c'est-à-dire dans le cas présent l'ensemble mer Rouge / golfe Persique / mer $\mathrm{d}^{\prime} O \mathrm{man}^{63}$ ), à savoir des plantes basses émergées mais aussi, et surtout, des arbres : «Au large de nos côtes, toute la végétation est de petite taille (...) tandis que là-bas [i.e. dans la mer Extérieure] (...) il y en a aussi d'autres plus hautes qui sont des arbres ${ }^{64} \gg$. Bien qu'il ne le dise pas ouvertement ${ }^{65}$, Théophraste est certainement convaincu (comme sans doute les compagnons d'Alexandre) que seuls les rivages de la mer Extérieure, situés dans des régions particulièrement favorisées par l'activité solaire, pouvaient produire des plantes arborescentes plus ou moins spectaculaires. Il assumait l'héritage de la science ionienne, qui avait depuis longtemps réfléchi à l'influence des conditions climatiques sur les productions naturelles. Théophraste reconnaît donc dans cette strate végétale une formation propre aux zones littorales chaudes et, s'il n'a pas inventé le terme « mangrove », il en a partiellement formulé le concept ${ }^{66}$.

Par ailleurs, Théophraste s'est intéressé à des questions de physiologie végétale. Certaines réflexions - il est toutefois possible qu'elles émanent des auteurs qu'il utilise - montrent qu'il s'est interrogé sur la nature exacte du milieu (ôiżüió) dans lequel se développe la mangrove. Théophraste semble proposer deux réponses, qu'il n'a pas explicitement cherché à accorder, mais qui ne se contredisent pas. Dans sa classification, il avait rangé ces arbres dans la catégorie des plantes d'eau salée. Aussi trouve-t-on à plusieurs reprises cette idée, que la mer constitue le biotope de la mangrove : «Il existe en effet des plantes (...) qui poussent jusque dans la mer (...) dont les plus grandes se trouvent sur les bords de la mer Érythrée »; « C'est aussi dans la mer que poussent certaines plantes ligneuses et même, dans la mer Extérieure, des arbres. (...) Là-bas même les espèces de ce type [sc. les plantes ligneuses] sont émergées, et il y en a aussi d'autres plus hautes qui sont des arbres»; «il y a, dans la mer même, des arbres $(\ldots) »^{68}$. Le propos ne souffre aucune ambiguïté lorsque Théophraste oppose la mangrove à la flore terrestre : « (...) sur terre aucun arbre ne pousse (...). Dans la mer, au contraire, poussent des arbres ». Cependant, dans un passage d'interprétation difficile, au sujet de « petits » végétaux ${ }^{69}$ marins immergés (des plantules d'arbres de mangrove $^{70}$ ? des algues ? des plantes basses submergées ?), Théophraste s'exprime différemment. En effet, distinguant les arbres de mangrove, portant feuilles, fleurs et fruits, de cette strate végétale basse, il explique que ceux-ci poussent « sur la terre que recouvrent les

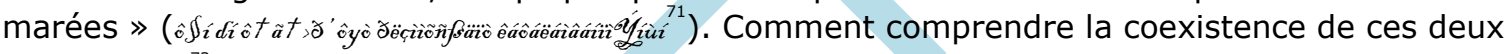
affirmations ${ }^{72}$ ? Soit la seconde, plus précise, affine la première, plus générale, en spécifiant la nature amphibie de la mangrove (terrestre parce qu'elle s'ancre dans un substrat terreux, marine parce qu'elle subit constamment l'inondation du flux) ; soit Théophraste veut distinguer deux

61 Théophraste, H. P., 4, 1, 5 (trad. S. Amigues).

62 Théophraste, H.P., 4, 6, 1. Noter que la mangrove est bien distinguée des végétaux terrestres qui supportent occasionnellement l'eau salée, comme le palmier, la scille ou l'asphodèle, ou qui recherchent un milieu saumâtre (Théophraste, H. P., 1, 4, 3 ; 2, 6, 2-3 ; C. P., 2, 5 ; voir S. Amigues, Études, p. 5.)

63 En ce qui concerne la mangrove, Théophraste assimile mer Extérieure et mer Érythrée.

64 Théophraste, H. P., 4, 6, 1 (traduction S. Amigues) ; cf. aussi Idem, H. P., 1, 4, 2. L'organisation du livre 4 reflète cette classification.

65 Voir cependant Théophraste, H. P., 1, 7, 1.

66 Cf. WMA, p. 19 : «The term « mangrove » has been used variously to define a constituent group of plants and the communities in which these occur »; P.B. Tomlinson, Mangroves, p. 3 : « Mangroves in the more limited sense may thus be defined as tropical trees restricted to intertidal and adjacent communities ». Le mot serait né au $\mathrm{XVI} \mathrm{I}^{\mathrm{e}}$ siècle.

67 C'est le mot qu'utilise Théophraste pour désigner l'habitat d'une plante (voir H. P., 1, 4, $2 ; 1,4,4$ ).

68 Théophraste, H.P., 1, 4, 2 ; 4, 6, 1 ; 4, 7, 5. Cf. aussi Id., H. P., 1, 13, 1 ; 4, 7, 2 ; 4, 7, 8.

69 C'est-à-dire, probablement, qui n'ont pas une hauteur et un port permettant de les classer dans la catégorie des arbres. Cf. Théophraste, C. P., 2, 5, 2-5.

70 Interprétation de H. Bretzl, B.F., p. 258, critiquée par Ch. Joret, Les recherches botaniques, p. 501. Voir aussi S. Amigues, Recherches, t. 1, p. 258, n. 20.

71 Théophraste, H.P., 4, 7, 6. Voir aussi Id., H. P., 4, 7, 4 : «Dans les îles recouvertes par la marée poussent de grands arbres (...)».

72 On en retrouve un autre exemple dans Théophraste, C. $P ., 2,5,2$. 
milieux : celui d'une mangrove toujours immergée et celui d'une mangrove alternativement couverte et découverte ${ }^{73}$.

Théophraste discute aussi du problème de la survie en milieu salé. Au reste, cette question - comme d'autres d'ailleurs- a dû faire l'objet de débats parmi les compagnons d'Alexandre. On le

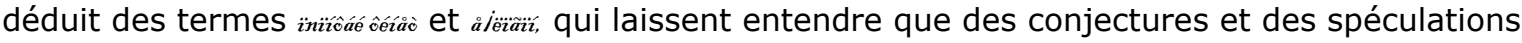
avaient circulé ${ }^{74}$. Repartons, pour comprendre les réflexions de Théophraste, de la bipartition évoquée ci-dessus (végétation de taille réduite / arbres). Il semble que pour cette végétation basse immergée, non identifiable pour nous, il n'y avait guère de doute : ces plantes étaient adaptées à I'eau salée (on faisait sans doute I'analogie avec les banales algues que tout Grec connaissait ${ }^{75}$ ). Le véritable problème concernait les arbres de la mangrove : comment des végétaux en tout point analogues à leurs congénères terrestres pouvaient-ils résister à l'eau salée qui les inondait et baignait leurs racines ? Deux hypothèses apparaissent chez Théophraste. La première dut être forgée quand les observateurs constatèrent que, dans le golfe Persique oriental, des arbres poussaient dans l'eau salée sans que l'on puisse détecter aux alentours la moindre trace d'eau douce $^{76}$ : ces arbres devaient « se nourrir » d'eau de mer, à la façon des plantes strictement marines, telles les algues ${ }^{77}$, à ceci près qu'ils poussaient plus haut, portaient des feuilles, fleurissaient et fructifiaient. Mais il semble bien que cette hypothèse, qui contredisait l'expérience ordinaire de tout homme qui cultive la terre, était difficile à accepter ${ }^{78}$. Une autre conclusion semble emporter la préférence de Théophraste, peut-être parce qu'elle ne heurtait ni le bon sens ni I'expérience : les racines prélevaient (on ne sait comment) de l'eau douce dans la terre, cependant que l'eau de mer n'abîmait pas la plante ${ }^{79}$. Quoiqu'il en soit de ces difficultés théoriques, nul, dans I'Antiquité, n'a pu dépasser la réflexion de Théophraste.

\section{3) Dégradation des connaissances après l'époque hellénistique : Pline et Plutarque}

Dès le II ${ }^{\mathrm{e}}$ siècle av. J.-C., comme cela a été dit plus haut, les mangroves ne font guère l'objet de recherches et paraissent attirer faiblement la curiosité du public cultivé. La meilleure somme de connaissances post-hellénistique demeure, dans l'état actuel de la documentation, I'Histoire naturelle de Pline. Mais $\mathrm{H}$. Bretzl a bien montré que cette collection de remarques éparses dépend largement de Théophraste et ne l'égale pas ${ }^{80}$. Il est donc inutile de revenir sur ce point. Le reste des connaissances d'époque romaine se réduit, ou peu s'en faut, à deux remarques de Plutarque. On y découvre un savoir de seconde main, dépourvu de description, de réflexion, de curiosité ; la mangrove n'est pas examinée pour elle-même mais est mentionnée brièvement, à titre d'exemple, pour appuyer un argument.

La première occurence se trouve dans les Questions naturelles. À la question "Pourquoi l'eau de mer ne nourrit-elle pas les arbres [terrestres]?"81, Plutarque propose plusieurs réponses argumentées : soit la densité de l'eau de mer ne lui permet pas de pénétrer dans les racines ${ }^{82}$; soit la salure dessèche l'arbre, agissant comme le sel sur un cadavre; soit elle lui nuit par sa nature "graisseuse ${ }^{\mu^{83}}$; soit enfin, l'eau salée est impropre à nourrir les arbres parce qu'elle contient un

\footnotetext{
73 Aristobule (Arrien, Anab., 6, 22, 6-7) faisait une distinction de ce type.

74 Théophraste, $H$. $P$. , 4, 7, 6. Un autre passage de Théophraste (C. $P ., 2,5,2)$ peut également laisser entendre que c'était une question débattue.

75 Théophraste, H.P., 4, 7, 6 ; De caus. plant, 5, 2, 5.

76 Théophraste, $H . P ., 4,7,6$. Nous comprenons ce passage ainsi : «Il n'y a absolument pas d'eau [douce] dans cette région. Il reste bien des canaux sur lesquels on navigue, mais ils contiennent de l'eau de mer. De ce fait, certains croient évident que [ces arbres] se nourrissent d'eau de mer et non d'eau douce, à moins qu'ils ne tirent quelque chose (i.e. en eau douce) de la terre par leurs racines; mais il est plausible que cela (i.e. ce qu'ils tirent) soit également saumâtre, car les racines ne sont même pas profondes ».

77 C'est pourquoi (cf. Théophraste, $H . P ., 4,7,6$ ) on pouvait conclure que ces arbres et ces plantes basses

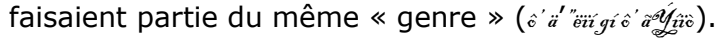

78 Pour Bretzl, B.F., p. 65, Théophraste penche pour une «nourriture » d'eau de mer. Pline, 12, 37, admet aussi cette idée. Voir également Théophraste, C. P., 2, 6, 3.

79 Hypothèse clairement formulée dans Théophraste, C. $P$., 2, 5, 2 et présente dans Id., H. P., 4, 7, 6 (cf. supra, n. 76). On peut signaler à ce sujet que la science grecque admettait que l'eau de mer pût contenir de l'eau douce (cf. Aristote, Meteor., 2, 3 ; H. A., 8, 2).

80 H. Bretzl, B.F., p. 81-95.

81 Plutarque, Quaest. Nat. 1 (Mor., 911F).

82 Sur la densité de l'eau salée cf. Aristote, Probl., 23, 7 ; 23, 10 ; Meteor., 2, 3, 359a5.

83 Cf. Aristote, Probl., 23, 7.
} 
élément "terreux" ${ }^{\prime 84}$. Intervient alors la remarque suivante : "Les arbres et les plantes, dont on dit qu'ils croissent dans la mer Érythrée, ne portent aucun fruit et sont nourris par les fleuves qui charrient une grande quantité de limon. C'est pourquoi ils poussent non pas devant la terre (i.e. dans la pleine mer ?), mais à son voisinage". Cette référence à la mangrove est obscure. Plutarque, semble-t-il, veut conclure son chapitre en montrant que les arbres de l'Érythrée sont nourris par de l'eau douce, même s'ils paraissent pousser dans l'eau de mer, et qu'ils ne constituent donc pas une exception végétale ; cette eau douce proviendrait des cours d'eau, qui apporteraient en même temps un substrat terreux ${ }^{85}$. Soit Plutarque exprime une réflexion personnelle, soit il reprend à son compte une thèse antérieure, aujourd'hui disparue, dont l'auteur devait sans doute discuter du biotope de la mangrove. Reste que le propos de Plutarque dénote une dégradation du savoir : aucun observateur hellénistique sérieux n'a écrit que ces arbres ne fructifiaient pas, bien au contraire (c'est un de leurs traits caractéristiques). Par ailleurs, dans l'état actuel de la documentation, aucun observateur antique n'a spécifié la relation entre fleuves et mangroves; le corpus documentaire atteste que, tant pour les spécialistes que pour l'opinion commune, la mangrove appartient au domaine marin ${ }^{86}$. De plus, la quasi-totalité des mangroves observées dans I'Antiquité poussent sur des littoraux désertiques, privés de cours d'eau. On se demande même si

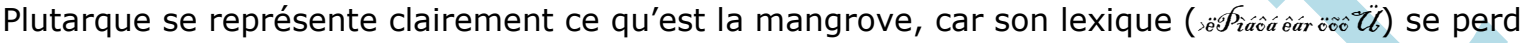
dans le flou. Tout ceci donne à penser qu'il utilise assez maladroitement, dans un propos technique, l'exemple d'une réalité qu'il a médiocrement comprise.

Dans le traité De facie in orbe Lunae, un personnage de Plutarque évoque, pour les besoins de son argumentation ${ }^{87}$, les mangroves de Troglodytique (rive occidentale de la mer Rouge et corne africaine) et de Gédrosie : « Alors que la Gédrosie et la Troglodytique qui s'étend le long de l'océan sont entièrement stériles et désertes, en raison de la sécheresse, dans la mer bordière qui les baigne croissent des plantes d'une étonnante grandeur, qui poussent en eau profonde ( ou « sous l'eau »?) : on les appelle « oliviers », «lauriers », « chevelure d'Isis» (trad. partiellement empruntée à S. Amigues). En apparence, Plutarque apporte du nouveau : d'une part il regroupe la Troglodytique et la Gédrosie, d'autre part il leur attribue des arbres semblables; en effet, d'après les sources antérieures, le laurier et I'olivier se trouvent en mer Rouge et non en mer d'Oman. En réalité, cette information, faussement inédite, résulte d'une compilation artificielle de données ${ }^{88}$. Le contraste entre la sécheresse de la terre ferme et la végétation foisonnante de la mer, l'immersion des arbres, l'espèce dite "chevelure $\mathrm{d}^{\prime} \mathrm{Isis}{ }^{89}$ étaient connus dès l'époque hellénistique, pour la mer Rouge comme pour la mer d'Oman ; il suffisait à Plutarque de tout regrouper autour de I'association sectorielle Troglodytique-Gédrosie. Quant aux espèces dénommées "olivier" et "laurier", il semble bien qu'Ératosthène avait, dès le III ${ }^{\mathrm{e}}$ siècle, étendu par généralisation à la totalité du littoral de l'Érythrée (c'est-à-dire à l'ensemble mer Rouge / golfe Persique / mer d'Oman) ces arbres connus jusqu'alors pour la seule mer Rouge ${ }^{90}$. Plutarque, simple compilateur,

\footnotetext{
84 Emprunt explicite à Aristote, Meteor., 2, 3, 357a-359b : I'eau salée est définie comme un mélange d'eau douce et d'un résidu « terreux » issu d'une action du feu.

85 Plutarque s'appuie peut-être encore sur Aristote, qui observe que, le plus souvent, les eaux marines du littoral sont plus douces et moins saumâtres que celles du large (Aristote, Probl., 23, 19).

86 Il est donc fort douteux que Plutarque, comme le croit H. Bretzl, B.F., p. 67, ait connu l'existence des mangroves fluviatiles, manifestant ainsi le progrès des connaissances antiques. La spécificité des mangroves fluviatiles échappait complètement au savoir des Anciens.

87 Plutarque, De facie in orbe Lunae, 25 Le locuteur fait l'inventaire de certaines particularités du règne végétal pour montrer qu'il est concevable que des plantes poussent sur la lune.

88 Plutarque peut en être l'auteur mais elle peut remonter à une source plus ancienne.

89 Cf. Théophraste, H. P., 4, 7, 3 (cependant ne sont pas nommés « chevelure d'Isis ») ; Photius, 460a (= Agatharchide, Érythr., 108); Pline, 13, 142 (chevelure d'Isis dans des îles des Trogodytes, d'après Juba de Maurétanie).

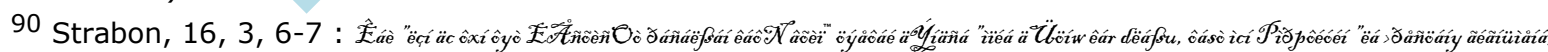

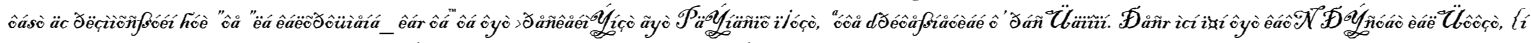

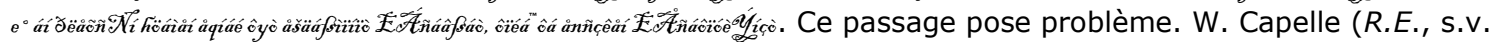

Nearchos, 2147-2148) et H. Strasburger (Alexanders Zug, p. 462-463, n. 2) ont jugé qu'Ératosthène décrivait seulement les mangroves du golfe Persique et qu'il empruntait ses renseignements à Néarque ou Androsthène. Ceci ne nous paraît pas convaincant. En effet, comment imaginer que les navigateurs d'Alexandre aient nommé des arbres que le plus méticuleux et le plus précis des botanistes antiques, Théophraste, aurait ignorés? À notre avis, bien que cette remarque d'Ératosthène prenne place dans un exposé que Strabon a voulu consacrer au golfe Persique, elle ne se limite pas à ce secteur. En effet, quand Ératosthène utilise le nom Érythrée (a fortiori s'il est renforcé par "ë́i)), celui-ci renvoie clairement à l'ensemble mer d'Oman / golfe Persique /mer Rouge (cf. Strabon, 16, 3, 1; G. Aujac, Strabon, p. 288), alors que les deux golfes sont désignés par leur appellation respective (cf. Strabon 17, 1, 1; 17, 3, 2). Le savant a vraisemblablement étendu à tout le domaine érythréen les oliviers et lauriers du golfe Arabique, non par négligence, mais par
} 
n'a donc pas fait autre chose que reprendre et compiler des données anciennes, au prix de l'approximation et de l'inexactitude ${ }^{91}$.

\section{III) Les espèces reconnues et leur description}

\section{1) Les espèces décrites dans l'Antiquité}

Remarque préliminaire : ne sont ici pris en compte que les témoignages utilisables dans le cadre d'une identification botanique. Ils seront répertoriés dans la rubrique testimonia.

1.1) L'arbre « au fruit semblable à l'amande »

- Testimonia : Théophraste, H.P. 4, 7, 5 (Perse située du côté/ en direction de la Carmanie [partie littorale]) ; Pline, 12, 37 (Perse ; golfe Persique)

- Identification selon H. Bretzl et S. Amigues ${ }^{92}$ : Avicennia officinalis L. / A. marina (Forsk.) Viehr. ${ }^{93}$

- Critères d'identification sûrs :

a) Fruits abondants et semblables à l'amande. Pline, on ne sait pourquoi, établit une ressemblance externe complète entre les deux fruits, alors que Théophraste se limite à la couleur extérieure $^{94}$. L'informateur de Théophraste s'en tenait peut-être à ce seul détail s'il observait $A$. marina. Le fruit de cette espèce, vert et duveteux, n'a pas une forme strictement amygdoïdale (« nearly spherical to slightly ovoid in outline but is always about as long as it is wide... ; the short beak ... is almost completely obscure in the mature fruit $\left.^{95} \gg\right)$. Au demeurant, si I'on s'en remet aux données disponibles actuellement, Avicennia marina conviendrait mieux aux témoignages anciens : cette espèce occupe aujourd'hui un vaste espace, depuis I'Afrique orientale et la mer Rouge jusqu'à la mer de Chine méridionale, tandis que Avicennia officinalis L. ne dépasse pas à l'ouest les côtes méridionales de l'Inde et ne se trouve donc pas dans la région du golfe Persique ${ }^{96}$.

b) Organisation interne du fruit. L'observateur dont Théophraste rapporte le témoignage indique qu'à l'intérieur, le fruit est « pelotonné, comme s'il était comprimé de toutes

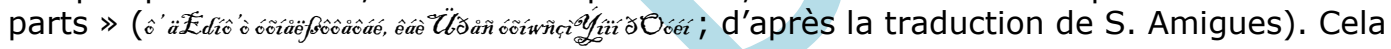
convient au genre Avicennia : «Il renferme une graine formée deux gros cotylédons souvent inégaux ; ceux-ci ont la particularité, bien notée par l'observateur de Théophraste, d'être comprimés et plissés longitudinalement ${ }^{97} \gg$. P.B. Tomlinson écrit pour sa part : « The cotyledons are thick (to $3 \mathrm{~mm}$ ), fleshy, and folded in opposite directions. The cotyledons also may differ appreciabily in shape ; the outer is broadly emarginate or even bilobed, and

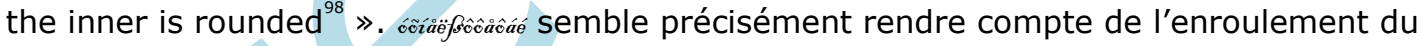
cotylédon extérieur qui enserre le cotylédon intérieur ${ }^{99}$; cette organisation interne peut donc justement donner l'impression à l'observateur ouvrant le fruit que celui-ci paraît «comprimé de toutes parts ». Pline donne moins de détails et utilise le mot « noyau » (intus contortis nucleis) ${ }^{100}$.

- Critères d'identification incertains :

généralisation théorique : si de tels arbres poussent sur les rivages africains de la mer Rouge, alors les mêmes doivent exister sur le reste du littoral érythréen, qui se trouve sous le même "climat".

91 Contra, cf. S. Amigues, Recherches, t. 2, p. 248.

92 H. Bretzl, B.F., p. 41-47 ; S. Amigues, Recherches, t. 2, p. 250 ; p. 258.

93 P.B. Tomlinson, Mangroves, p. 199-200, distingue clairement les deux espèces. Cependant, des confusions se sont produites (le nom Avicennia officinalis a été parfois improprement attribué à Avicennia marina) : "The ultimate [confusion] is the reduction of all species and varieties under this one name $[A$. officinalis] ». S. Amigues, Recherches, t. 2, p. 248 considère que le nom $A$. marina remplace $A$. officinalis.

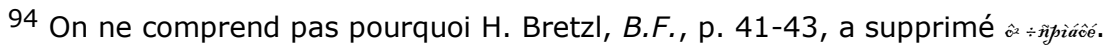

95 P. B. Tomlinson, Mangroves, p. 201.

96 P. B. Tomlinson, Mangroves, p. 199 et p. 201 ; WMA, cartes p. 32 et 34 ; p. 158.

97 S. Amigues, Recherches, t. 2, p. 258.

98 P. B. Tomlinson, Mangroves, p. 195.

99 Voir H. Bretzl, B.F., p. 42, illustrations n०2 et $\mathrm{n}^{\circ} 4$; P.B. Tomlinson, Mangroves, p. $189, \mathrm{n}^{\circ} 8 \mathrm{j}(A$. germinans) et p. $203 \mathrm{n}^{\circ} \mathrm{B} 14$ ( $A$. alba).

100 Cf. H. Bretzl, B.F., p. 84. 


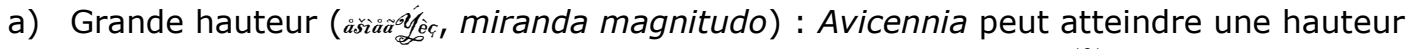
respectable mais ce critère relève d'une appréciation subjective ${ }^{101}$.

b) Aspect (ïй̈̈t, specie) de l'arbousier d'Orient (Arbutus andrachne). Ceci n'est pas invraisemblable. Le port dense des sujets d'Avicennia jeunes, la présence de grappes de fruits arrondis, verts peut rappeler l'arbousier.

c) Feuille semblable à celle de l'arbousier d'Orient. Théophraste (Pline ne dit rien à ce sujet) ne précise pas sur quel élément porte la ressemblance : forme, couleur ou texture. Des différences réelles opposent les deux feuilles (celle de l'arbousier est vert clair, glabre à la face inférieure). Toutefois, comme l'ont remarqué $\mathrm{H}$. Bretzl et $\mathrm{S}$. Amigues, les feuilles d'A. (officinalis ou marina) sont si variables dans leur forme (selon la zone d'origine ou le degré de croissance de la feuille) et dans leur aspect (présence plus ou moins prononcée de duvet) que le jugement de l'observateur antique est acceptable ${ }^{102}$.

- Donnée problématique : racines-échasses. Elles caractérisent le genre Rhizophora et non Avicennia, qui est pourvu de pneumatophores. S. Amigues admet une erreur de la part de I'observateur antique ${ }^{103}$. Les causes de celles-ci nous échappent d'autant plus que l'observateur semble avoir approché l'arbre (puisqu'il en a ouvert le fruit). Cependant certaines espèces ( $A$. officinalis plus que $A$. marina) émettent des racines aériennes pendantes, semblables à des racines-échasses quand, parfois, elles se développent à partir des branches basses ${ }^{104}$. Il n'est cependant pas certain que ceci suffise à sauver cette partie du témoignage antique.

2.2) L'arbre « à fleur de violier »

- Testimonia : Théophraste, H.P. 4, 7, 4 (dans des îles [de la mer parcourue par l'expédition de retour de I'Inde] recouvertes par la marée ${ }^{105}$ ); Pline, 13, 141 (îles de la mer parcourue par I'expédition de retour de I'Inde) ; Arrien [Aristobule], Anab., 6, 22, 6-7 (Gédrosie) ${ }^{106}$

- Identification selon H. Bretzl (mais cf. ci-dessous) et S. Amigues : Rhizophora mucronata Lamk. ${ }^{107}$

Remarque : discutant du paragraphe précité de Théophraste, $\mathrm{H}$. Bretzl estime qu'il y est en réalité question de deux espèces différentes : les arbres « aussi hauts que les plus grands platanes et peupliers » seraient, selon lui, des Avicennia, tandis de ceux dont les racines et les sommets peuvent servir de point d'attache aux amarres, qui ont une fleur semblable à celle du violier blanc etc. seraient des Rhizophora ${ }^{108}$. S. Amigues, à juste titre, refuse cette distinction ${ }^{109}$ : Théophraste parle bien ici d'une seule et même espèce ( $R h$. mucronata). On ajoutera à ses arguments : 1 ) que l'analyse interne du passage de Théophraste plaide en ce sens : la possibilité d'accrocher les amarres au sommet ou au pied des arbres, selon les marées, suit logiquement l'observation sur leur hauteur remarquable ; 2) que Théophraste utilise généralement l'adjectif đë̈ä quand il décrit un autre arbre, ce qui n'est pas le cas ici ; 3) que le texte de Pline, qui démarque Théophraste, corrobore cette hypothèse.

- Critères d'identification sûrs :

a) Racines. Les racines auxquelles peuvent être accrochés des cordages de navires sont, comme l'a vu $\mathrm{H}$. Bretzl, des racines-échasses, caractéristiques des Rhizophoracées ${ }^{10}$.

b) Fleur semblable à celle du violier blanc. $R h$. mucronata produit, au-dessus d'un calice étalé et jaune, une fleur à quatre pétales, blancs ou blanc-vert ou jaune, couleurs qui

101 Cf. infra, p. .

102 H. Bretzl, B.F., p. 43-44 ; S. Amigues, Recherches, t. 2, p. 257-258. P.B. Tomlinson, loc. cit. n. 98, présente des figures montrant clairement la variabilité des feuilles d'Avicennia. Plus problématique est la question du duvet : d'après le même auteur, p. 82 , la présence de duvet semble être caractéristique du genre Avicennia.

103 S. Amigues, Recherches, t. 2, p. 219.

104 P.B. Tomlinson, Mangroves, p. 97-99 et p. 195.

105 Cette localisation géographique est trop imprécise pour en tirer quelque conclusion que ce soit, en particulier que l'aire de Rhizophora mucronata atteignait dans I'Antiquité le golfe Persique (S. Amigues, Recherches, t. 2, p. 258-259; Études, p. 272-274).

106 H. Bretzl, B.F., p. 64, sur le seul critère du parfum de la fleur, identifie l'arbre que décrit Arrien (Aristobule) à Aegiceras corniculatum.

107 Il semblerait que Rh mucronata atteigne encore aujourd'hui les limites du golfe Persique (P. B. Tomlinson, Mangroves, p. 331).

108 H. Bretzl, B.F., p. 45 et p. 57-58 : pour l'auteur, Rh. mucronata ne dépasse pas deux mètres et ne peut être qualifié d'arbre élevé (à la différence d'A. officinalis).

109 S. Amigues, Recherches, t. 2, p. 256.

110 H. Bretzl, B.F., p. 57. 
correspondent à celle de Matthiola incana, le leukon ion des Grecs ${ }^{111}$ (S. Amigues précise que la comparaison, chez les descripteurs antiques, ne se fonde que sur la couleur et le parfum ; il n'est pas tenu compte du nombre des pétales ${ }^{112}$ ).

c) Fruit semblable à l'olive : « un fruit ovale allongé en forme d'olive est caractéristique des mangliers ${ }^{113} \gg$. Voir cependant ci-après (《 développement du fruit »).

- Critères d'identification incertains : feuille semblable à celle du laurier. Les feuilles de $R h$. mucronata, simples, entières, elliptiques, la face inférieure piquetée de stomates, se caractérisent par leur grande taille (10 à $20 \mathrm{~cm}$ de long, 4 à 10 de large), leur bord recourbé, leur aspect brillant, avec leur « apex» (《mucro »), caractéristique, à l'extrémité (il est néanmoins souvent brisé $)^{114}$. La ressemblance avec les feuilles de laurier $n^{\prime}$ est que très partielle et il est difficile de qualifier, comme le fait $\mathrm{H}$. Bretzl, la feuille de $R h$. mucronata de lancéolée, alors qu'elle paraît elliptique (il suffit de se reporter à l'illustration qu'il propose) ${ }^{115}$.

- Données problématiques:

a) Développement du fruit. Comme d'autres espèces de mangrove, Rh. mucronata est vivipare : la graine se développe sur l'arbre, émettant un long hypocotyle qui peut atteindre $60 \mathrm{~cm}$; le fruit se détache seulement à ce stade. H. Bretzl ${ }^{116}$ a bien vu les aberrations de la description antique à ce sujet. En effet, l'observateur auquel se réfère Théophraste affirme que le fruit (mûr) tombe au printemps. Or à ce moment précis, d'une part le fruit ne ressemble plus du tout à une olive ; d'autre part les navigateurs se trouvaient en dehors de la zone où pousse Rh. Mucronata. Outre ceci, le texte de Théophraste n'est pas clair, dans la mesure où il laisse entendre que la fleur et le fruit se forment simultanément à l'automne et tombent ensemble au printemps. Aristobule est plus exact ou plus prudent que l'observateur qu'utilise Théophraste : paraissant s'en tenir à ce qu'il a vu personnellement, il se contente de dire qu'il a aperçu l'arbre en fleurs lors de son passage, c'est-à-dire en automne ; à la différence de l'observateur de Théophraste, il a vu la floraison mais pas la fructification.

b) Hauteur des arbres. D'après les spécialistes de la mangrove, le genre Rhizophora (comme le genre Avicennia) peuvent, selon les conditions naturelles, donner soit de véritables arbres et former une véritable canopée, soit rester au stade d'arbrisseau ${ }^{117}$. Ainsi, d'après les données contemporaines, certaines espèces de Rhizophora comptent des individus pouvant atteindre $30 \mathrm{~m}$. , tandis que $R h$. mucronata peut s'élever à plus de $15 \mathrm{~m}^{118}$. Aristobule, signalant que, dans une mangrove de la côte gédrosienne, des arbres atteignent parfois 30 coudées (13 m.), rapporte donc des données très acceptables. En revanche, comme l'a remarqué $\mathrm{S}$. Amigues, celui qui a renseigné Théophraste se trompe ou exagère lorsqu'il confronte la hauteur des arbres et le niveau des marées : il est invraisemblable qu'un arbre de la hauteur d'un très grand peuplier ou d'un très grand platane soit presque entièrement recouvert au flux pour être ensuite presque à sec au reflux, les racines-échasses apparentes : de la côte occidentale de I'Inde jusqu'au golfe Persique, les amplitudes moyennes de marée ne dépassent pas $5 \mathrm{~m} .{ }^{119}$. L'une ou l'autre des données n'est pas admissible (une fois encore, Aristobule, plus précis et plus exact, n'affirme pas que les plus hauts arbres sont presque recouverts par la mer).

2.3) L'arbre «à fruit de lupin »

- Testimonia : Théophraste, H.P. 4, 7, 5 (mer parcourue par l'expédition de retour de I'Inde) ; 4, 7, 6 (Perse située du côté/ en direction de la Carmanie [partie littorale]) ; 4, 7, 7 (Tylos) ; Pline, H.N., 12, 38 (Tylos) ; 13, 141 (mer parcourue par l'expédition de retour de l'Inde)

- Identification selon H. Bretzl et S. Amigues : Aegiceras majus Gaertn. (= A. corniculatum (L.) Blanco) ${ }^{12}$

111 P.B. Tomlinson, Mangroves, p. 327 (« white or greenish white ») ; cf. aussi H. Bretzl, B.F., p. 58 (« candidi postea tamen rubescentes »).

112 S. Amigues, Recherches, t. 2, p. 256. Sur le nombre de pétales, cf. H. Bretzl, B.F., p. 58.

113 S. Amigues, Recherches, t. 2, p. 250.

114 P. B. Tomlinson, Mangroves, p. 326-327.

115 H. Bretzl, B.F., p.56-57 ; S. Amigues, Recherches, p. 250.

116 H. Bretzl, B.F., p. 59-60.

117 P. B. Tomlinson, Mangroves, p. 65.

118 P.B. Tomlinson, Mangroves, p. 326.

119 S. Amigues, Recherches, t. 2, p. 257 ; WMA, p. 53 ; 63 ; 159.

120 N.b. : voir supra, n. 106. 
- Critères d'identification sûrs :

a) Fruit. Il a en effet une forme tout à fait caractéristique qui justifie pleinement la comparaison établie par les descripteurs antiques. Toutefois on comprend mal ce qui rend le fruit non comestible (dureté ? âpreté ? sel ${ }^{121}$ ? toxicité ?).

b) Parfum agréable de la fleur, qui a valu à cette plante d'être aussi nommée $A$. fragrans ${ }^{122}$ (il faut admettre que les Gréco-Macédoniens ont pu voir simultanément fleurs et fruits).

- Données problématiques :

a) Aire de répartition (Théophraste, H.P., 4, 7, 7 ; Pline, 12, 38) : Androsthène a aperçu $A$. majus à Bahrein, mais actuellement, dans I'hémisphère nord, cet arbre ne se trouve que dans I'Asie tropicale (de l'Inde et Ceylan à la Chine méridionale) ${ }^{123}$. Il faut donc admettre, en l'absence de tout autre information (seules des analyses palynologiques pourraient trancher), que Tylos serait le point le plus septentrional connu atteint par $A$. majus à l'époque d'Alexandre avant de disparaître pour des raisons inconnues ${ }^{124}:$ aujourd'hui, les mangroves du golfe Persique ne sont composées que d'Avicennia.

b) Densité des peuplements (à Tylos). D'après Androsthène, «il y a, sur la côte orientale, au

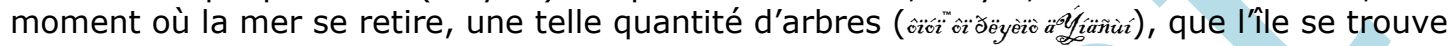

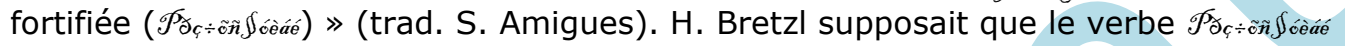
désignait métaphoriquement un rempart de pneumatophores d'Avicennia, émergeant de la vase ; il en déduisait donc la présence de deux espèces sur le littoral oriental de Bahrein : Aeg. majus et $A v$. officinalis ${ }^{125}$. S. Amigues reproche à $\mathrm{H}$. Bretzl de solliciter le sens du verbe grec ${ }^{126}$. On ajoutera à sa juste critique que Théophraste décrit explicitement la palissade que constituent, à marée basse, des peuplements serrés d'arbres et non de modestes pneumatophores. Il en résulte que, d'après les témoignages anciens, $A$. majus abondait à Bahrein au point de constituer des groupements importants qui donnaient I'image d'une barrière infranchissable. Or, d'après certaines informations contemporaines, A. majus ne se comporte pas ainsi : «It (...) occurs typically as an isolated low shrub, never forming a conspicuous part of the community ${ }^{127} \gg$. Soit il faut supposer, contre les données botaniques actuelles, l'existence d'une abondante forêt d'A. majus, qui constituait la mangrove frontale de Bahrein ; soit il faut supposer qu'Androsthène, victime d'une illusion d'observation, a vu «l'Aegiceras se détacher d'une masse de végétation indifférenciée et en réalité constituée d'Avicennia ${ }^{128} \gg$.

c) Racines échasses (Théophraste, H.P., 4, 7, 5 ; le problème n'apparaît plus chez Pline, qui a disloqué le texte de Théophraste). Le texte ambigu de Théophraste peut laisser entendre qu'A. majus possède un tel système racinaire, ce qui n'est pas le cas $^{129}$.

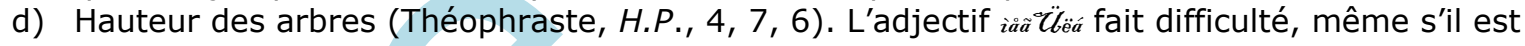
impossible de l'apprécier à sa juste valeur : $A$. majus, qui atteint au plus $6 \mathrm{~m} .$, s'apparente à un arbuste ${ }^{130}$. Soit les observateurs antiques ont réellement vu un $A$. majus mais ont jugé, à tort, que d'autres grands sujets qu'ils apercevaient (en arrière plan ?) appartenaient à la même espèce ; soit ces grands arbres à fruit de lupin ne sont pas des $A$. majus et il s'agit d'une autre espèce de mangrove. On peut, dans ce cas, se demander si I'hypocotyle de Rh. mucronata n'a pas été confondu, au premier stade de son développement, avec le fruit du lupin. Aussi cet arbre à fruit de lupin et fleurs parfumées pourrait-il être en réalité $R h$. mucronata, qui se couvre lui aussi d'une floraison odoriférante. Cela signifierait finalement que les Gréco-Macédoniens avaient réellement observé le phénomène de la viviparité, mais, incapables de la comprendre, l'avaient assimilé à une fructification.

121 Interprétation de H. Bretzl, B.F., p. 95.

122 H. Bretzl, B.F., p. 60-65 ; S. Amigues, Recherches, t. 2, p. 257 ; P. B. Tomlinson, Mangroves, p. 284-285.

123 P. B. Tomlinson, Mangroves, p. 27 ; p. 284 ; WMA, p. 31 (où il apparaît qu'Aeg. majus ne se trouve qu'à l'est du delta de I'Indus, mais, p. 44, l'espèce est mentionnée parmi celles qui se trouvent encore au Pakistan) ; p. 63 ; p. 158.

124 La prédation humaine n'est qu'un facteur parmi d'autres (cf. P.B. Tomlinson, Mangroves, p. 19-20).

125 H. Bretzl, B.F., p. 61-63. Ses conclusions sont suivies par D.T. Potts, The Arabian Gulf, t. 2, p.130-131.

126 S. Amigues, Recherches, t. 1, p. 259.

127 P. B. Tomlinson, Mangroves, p. 288 (contra, S. Amigues, Recherches, t. 2, p. 258).

128 S. Amigues, Recherches, t. 2, p. 259. Sur les erreurs d'observation, voir infra, p. .

129 Voir infra, p. .

130 P.B. Tomlinson, Mangroves, p. 285 
2.4) L' « olivier » et le « laurier » du golfe Arabique

À la différence des végétaux précédents, nous avons maintenant affaire à des arbres qui sont désignés par un nom précis et qui sont sont plusieurs fois associés dans les sources. Ils appartiennent à un secteur bien délimité : le golfe Arabique des Anciens (notre mer Rouge). Il est nécessaire, avant de progresser, de se pencher sur le témoignage capital de Théophraste, lequel,

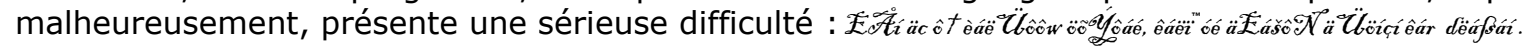

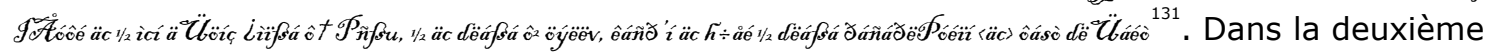

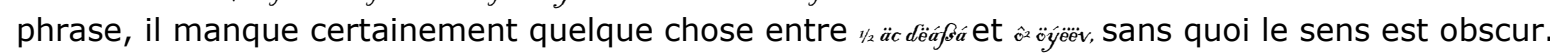

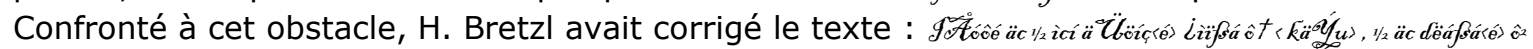
ï̌̈ërv. Procédant ainsi, il supposait et concluait que le laurier et l'olivier ne forment en réalité qu'une seule espèce : Avicennia (officinalis ?). Les variations de noms seraient dues à des différences d'aspect (« Formverschiedenheit ») ${ }^{132}:$ s'appuyant sur des descriptions arabes et contemporaines, $\mathrm{H}$. Bretzl estimait que l'Avicennia supporte la comparaison tant avec le laurier qu'avec l'olivier. Par ailleurs, le peuplement exclusif d'Avicennia en mer Rouge (au moment où il écrivait) confortait son point de vue.

La démonstration n'emporte pas la conviction. Tout d'abord, S. Amigues critique la sévérité des corrections ${ }^{133}$. Secondement, au $V^{\mathrm{e}}$ ou au IV $\mathrm{V}^{\mathrm{e}}$ siècle av. J.-C., d'autres espèces qu'Avicennia ont pu occuper les rivages de la mer Rouge. Troisièmement, Pline ${ }^{134}$, qui démarque Théophraste, ne laisse en aucun cas entendre que le laurier et l'olivier soient des arbres identiques : (...) in mari vero Rubro silvas virere, laurum maxume et olivam ferentis bacas (...). Enfin, dans les témoignages où le laurier et l'olivier sont mentionnés simultanément, la conjonction ne semble nullement

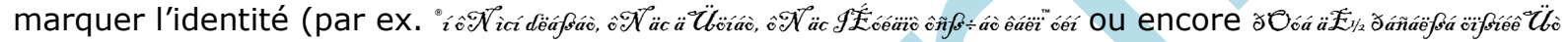

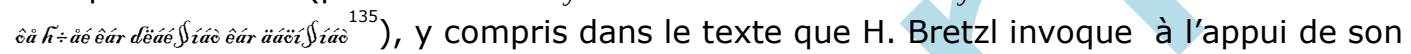

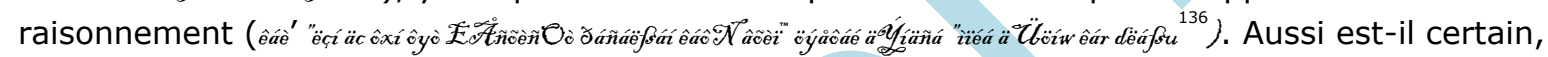
ou presque, que, par les noms « laurier » et «olivier », les Grecs désignaient deux arbres qu'ils jugeaient différents.

$\mathrm{L}^{\prime}$ « olivier 》

- Testimonia : voir ci-dessous.

- Identification : selon H. Bretzl, Avicennia officinalis dans tous les cas; selon S. Amigues, Rhizophora mucronata (mais n'écarte pas dans certains cas Avicennia marina).

- Critères d'identification (variables selon les témoignages) :

a) Fruit semblable à I'olive et/ou utilisation médicale (astringent) du suc de l'arbre (Théophraste, H.P., 4, 7, $2^{137}$ [dans la mer Érythrée -i.e. golfe Arabique- après Coptos] ; Pline, 13, 139 [même localisation]; Pline, 12,77 [Arabie $\left.{ }^{138}\right]$ ). Sur ces deux critères, $\mathrm{H}$. Bretzl identifiait I'olivier de Théophraste à $A$. officinalis. S. Amigues n'a pas manqué de relever le point faible de l'argumentation : Théophraste et Pline signalent une substance hémostatique, alors $\mathrm{H}$. Bretzl se réfère à une gomme odontalgique. En revanche $R h$. mucronata (dont le fruit avant germination vivipare ressemble à une olive ${ }^{139}$ ) sécrète un suc astringent, ce qui correspond mieux à l'arbre décrit par les Anciens ${ }^{140}$. Dans ce cas, le témoignage de Théophraste pourrait

131 Théophraste, H. P., 4, 7, 2.

132 H. Bretzl, B.F., p. 48-54.

133 S. Amigues, Recherches, t. 2, p. 249-251.

134 Pline, 13, 139.

135 Respectivement Plutarque, De facie in orbe Lunae, 25 et Strabon, 16, 4, 14, mais il suffit simplement de

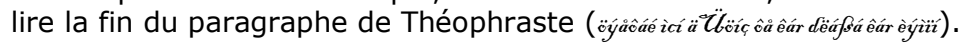

136 Strabon, 16, 3, 7 ; H. Bretzl, B.F., p. 53.

$137 \mathrm{~S}$. Amigues corrige le texte en rétablissant le mot « laurier » qui lui paraît le moins invraisemblable : 1/äc

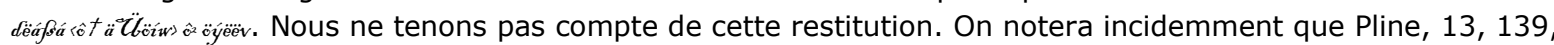
qui est assez proche de Théophraste, ne dit rien de la feuille de I'olivier.

138 Pline n'utilise pas directement Théophraste ici mais sans doute une source différente (ou intermédaire ?). Peut-être est ce Juba, qui est mentionné dans la liste des sources du livre 12). L'Arabie en question doit vraisemblablement être située sur la côte africaine du golfe Arabique, selon une terminologie courante dans I'Antiquité.

139 Cf. supra, p. .

140 H. Bretzl, B.F., p. 49-51 ; S. Amigues, Recherches, t. 2, p. 249-250. 
indiquer que I'aire de $R h$. mucronata atteignait, dans l'Antiquité, le littoral au débouché de la piste Coptos-mer Rouge, alors qu'aujourd'hui, I'espèce n'est signalée que dans l'État d'Érythrée ${ }^{141}$. Mais il convient d'être prudent : I'olivier en question semble être surtout connu pour le produit qu'il fournit, lequel fait sans doute l'objet d'un commerce. Or on sait qu'en ce domaine, les origines mentionnées ne sont jamais sûres.

b) Densité de peuplement (Photius, 456b = Agatharchide, Érythrée, 81 ; Diodore de Sicile, 3, 19, 3 [Agatharchide] ; 3, 39, 2 [Agatharchide] ${ }^{142}$. Agatharchide signale en mer Rouge (sur la côte des Ichtyophages et à proximité du port d'Aphrodite) des oliviers poussant en grand nombre. Ceci ne renseigne pas clairement sur l'espèce. La densité du peuplement est un caractère trop commun à divers arbres de mangrove pour autoriser une identification sérieuse (même si, au vu des données botaniques actuelles, Avicennia [marina], qui semble être l'espèce la plus adaptée aux conditions naturelles en mer Rouge et donc la plus apte à se développer en colonie, emporte la préférence ${ }^{143}$ ).

c) Fruit semblable à une châtaigne, de toute évidence sortie de sa bogue comme le laisse entendre le mot êẫúv (Photius, 450b = Agatharchide, Érythrée, 43 ; Diodore de Sicile, 3, 19, 3 [Agatharchide] - localisation : côte des Ichtyophages). Il n'est pas possible de se prononcer. Si I'on considère la forme générale, on peut pencher, non sans hésitation, pour le fruit d'Avicennia plutôt que pour celui de $R h$. mucronata : celui d'A. officinalis est long de $3 \mathrm{~cm}$, duveteux, plus long que large et présente une pointe courte à son extrémité ; la pointe du fruit d'A. marina, moins ovoïde, disparaît presque totalement à maturité ${ }^{144}$. Mais si l'on prend en compte la couleur, le fruit de $R h$. mucronata peut tout aussi bien convenir.

d) Olivier éthiopique à suc odontalgique (Strabon, 16, 4, 18 [Artémidore] ? ; Pline, 23, 72 ; Dioscoride, 1, 105, 6 ; Oribase, Coll. med., 14, 62 ${ }^{145}$ ). Si I'on admet que cet arbre est bien une espèce de mangrove, l'utilisation de sa gomme pour soigner les douleurs dentaires conduit à Avicennia $^{146}$. De fait, à cette latitude $\left(28^{\circ} \mathrm{N}\right.$. environ) on se trouve près de la limite septentrionale actuelle de la mangrove $\left(27^{\circ} \mathrm{N} .{ }^{147}\right.$ approximativement) ; les conditions naturelles dans ce secteur sont difficiles (salinité élevée, faible renouvellement des eaux, écarts de température, aridité côtièr ${ }^{148}$ ). Seule une mangrove constituée d'espèces tolérantes peut coloniser le littoral. Il paraît donc raisonnable de considérer que ces bois denses sont constitués d'Avicennia.

Le «laurier»

- Testimonia : Théophraste, H.P., 4, 7, 2 (seul texte exploitable)

- Identification : Avicennia officinalis / marina selon H. Bretzl et S. Amigues

- Critères d'identification (particulièrement faibles et incertains)

a) Feuille semblable à celle du chêne-vert. Sur ce critère, S. Amigues propose d'identifier le laurier à Avicennia.

b) Le nom « laurier ». D'après S. Amigues, les informateurs de Théophraste ${ }^{149}$ (Anaxicrate et ses compagnons) ont pu voir, à leur retour (date supposée : mars 324), des Avicennia marina en

\footnotetext{
141 WMA, p. 158. Toutefois, selon d'autres auteurs, une population très réduite subsiste de nos jours à l'extrême sud du littoral égyptien (Wet Coastal Ecosystems, p. 217 ; WMA, p. 32, carte 4.6). On peut penser que $R h$. mucronata constituait dans l'Antiquité des populations plus denses. En effet, cette espèce a du souffrir de la prédation humaine en raison des qualités de son bois. C'est ainsi qu'elle a disparu du Yémen (WMA, p. 29) et de I'Inde occidentale (F. Blasco ap. P.B. Tomlinson, Mangroves, p. 56).

142 On pourrait ajouter à cette collection Strabon, 16, 4, 18 (golfe Élanite) mais le texte n'est pas aussi explicite que les autres.

143 WMA, p. 162.

144 P. B. Tomlinson, Mangroves, p. 199-201.

145 Dans la mesure où Strabon nomme ces oliviers « éthiopiques », on est tenté de faire un rapprochement avec Dioscoride et Oribase. Cependant, Strabon ne mentionne que des vertus médicinales, sans autre précision. H. Bretzl, B.F., p. 55, rapprochait ce passage de Strabon de Photius 457b (= Agatharchide, Érythr., 91) et Diodore de Sicile, 3, 44, 3 [Agatharchide], mais cela ne suffit pas pour dire qu'Agatharchide connaissait la vertu odontalgique de I' « olivier ».

146 S. Amigues, Recherches, t. 2, p. 251, avec renvoi à Pline et Dioscoride ; H. Bretzl, B.F., p. 49-50.

147 En mer Rouge, la limite se trouve actuellement à $27^{\circ} 40^{\prime}$ N (WMA, p. 29 ; p. 158 [mangrove au débouché du golfe de Suez, côté Sinaï]) ou $27^{\circ} 14^{\prime}$ N (P.B. Tomlinson, Mangroves, p. 61 ; dans la baie de Myos Hormos). En tout état de cause, Avicennia reste le seul genre, ou presque, qui colonise les limites septentrionales de la mangrove (cf. V. Chapman, ap. S. Amigues, Études, p. 274).

148 WMA, p. 157-158; 162 ; P. B. Tomlinson, Mangroves, p. 61.

149 Voir nos réserves sur ce point, supra, p. .
} 
fleurs. Leur inflorescence peut rappeler celle du laurier, d'où cette désignation. Tout ceci est cependant extrêmement conjectural.

\section{2) Les limites de l'observation et de la description antiques}

Les observations antiques rassemblées ci-dessus offrent indiscutablement des détails précis et justes. Cependant, d'un strict point de vue botanique, aucune n'est entièrement satisfaisante, car aucune ne réunit, pour quelque espèce que ce soit, un ensemble cohérent et pertinent d'éléments descriptifs. L'information antique, quel que soit l'arbre considéré, reste frangée $\mathrm{d}^{\prime}$ incertitude. Ainsi, pour tel « arbre à fruit de $\operatorname{lupin}^{150}{ }^{150}$, on hésite entre Aegiceras majus et Rhizophora mucronata ; ou encore, on se heurte à l'incertitude du nom générique « olivier », dont il faut admettre qu'il ne désigne pas de façon rigoureuse et constante une espèce, ce qui amoindrit I'intérêt botanique de certains témoignages (que sont les « oliviers » du détroit de Bab el-Mandeb ? ou ceux du Limen Elaia ${ }^{151}$ ?).

Les faiblesses irréversibles des notices antiques découlent probablement des circonstances dans lesquelles la mangrove fut approchée et vue. Considérons le cas des navigateurs d'Alexandre, les plus attentifs des observateurs. Ils parcouraient, dans l'urgence, cet espace nouveau qui s'ouvrait à eux. Comme l'écrit Strabon, « ce qu'ils virent, ils en prirent connaissance au cours de déplacements militaires et au pas de course ${ }^{152} \gg$. Aussi la mangrove fut-elle sans doute observée de l'extérieur et non explorée de l'intérieur. Or il n'était guère aisé, depuis un bateau, dans un court laps de temps, de se livrer à un examen précis de la forêt et de ses arbres. Rares furent les moments où I'on eut, peut-être, le loisir de regarder plus attentivement : I'attente dans les bouches de I'Indus et la jonction avec Alexandre près de l'île de Keshm. Les explorateurs lagides ne paraissent pas avoir davantage stationné dans les mangroves, d'intérêt limité eu égard à leurs objectifs. Par ailleurs, face à ce type de paysage, la vision de l'observateur est aisément brouillée. L'enchevêtrement des arbres ne permet guère de les distinguer les uns des autres : ainsi, remarque $\mathrm{H}$. Bretzl, Avicennia se perçoit mal dans la barrière que forme au premier plan Rhizophora ${ }^{153}$. De surcroît, les principales espèces qui colonisent le littoral se ressemblent : les feuilles sont d'une couleur verte assez uniforme ; leur texture est plutôt coriace, comme il convient à des plantes qui, dans un milieu hostile, sont contraintes de limiter les pertes d'eau : « The rather dull, uninspiring and unchanging aspect of the mangal is largely due to the uniform leaf shape and texture of mangroves, coupled with the evergreen habit ${ }^{154} \gg$. La palette chromatique des fleurs, assez réduite, va du blanc au jaune. Il était donc difficile de distinguer clairement les différentes espèces d'un paysage qui, de plus, changeait en permanence sous l'action du flux et du reflux. On peut comprendre alors que toutes sortes d'arbres (I'arbre à fleur de violier, l'arbre au fruit semblable à celui du lupin, I'arbre au fruit semblable à l'amande ${ }^{155}$ ) aient paru à certains observateurs reposer sur des racines-échasses, ce qui est invraisemblable. C'est pourquoi aussi il n'est pas exclu, comme l'avait déjà justement pressenti $\mathrm{H}$. Breztl, que la spéculation théorique compensât les déficits d'observation : il est possible que I'un des compagnons d'Alexandre ait $a$ priori considéré, hors de tout examen, que le fruit de Rhizophora mucronata tombait à maturité au printemps ${ }^{156}$

Les procédés de description dont usent les Anciens portent en eux trois insuffisances consubstantielles, qui s'ajoutent aux difficultés de l'observation in situ. Premièrement, la description botanique antique ne suit aucune règle, puisqu'il n'existe ni taxinomie, ni nomenclature. En l'absence de principes régulateurs, les observateurs antiques composaient des notices subjectives, davantage destinées à évoquer qu'à décrire ${ }^{157}$. Aussi, dans ce paysage inconnu qu'était la mangrove, le voyageur ne relevait en général que les détails qu'il remarquait personnellement, et sans doute ceux qui lui paraissaient le plus singuliers ; si certains révèlent une observation

\footnotetext{
150 Théophraste, H. P., 4, 7, 6.

151 Respectivement, Photius, 460a (= Agatharchide, Érythr., 108) ; Strabon, 16, 4, 8.

152 Strabon, 15, 1, 2.

153 H. Bretzl, B.F., p. 45 ; p. 61 . L'auteur signale, p. 98, le cas d'un voyageur du XIX siècle qui, dans l'archipel Dahlak, ne sut plus faire la différence entre les genres Avicennia et Rhizophora. Sur le cas d'Aeg. majus, peut-être confondu avec Avicennia, voir supra, p. .

154 P.B. Tomlinson, Mangroves, p. 79 (certains clichés, par ex. 1.1, 1.2, 1.6 sont explicites)

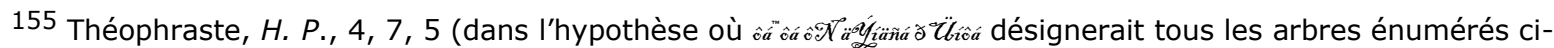
dessus).

156 H. Bretzl, B.F., p. 59.

157 Strabon, 15, 1, 2 : « C'est pourquoi ils ne rapportent pas non plus, sur des sujets identiques, les mêmes renseignements ».
} 
exacte (ainsi le fruit singulier d'Aegiceras majus), d'autres souffrent d'un relativisme sensoriel (la hauteur d'un arbre, voire la fragrance d'une fleur, sont des perceptions très relatives).

Deuxièmement, chez la plupart des descripteurs, la comparaison tient lieu de description (par exemple feuille « semblable à celle du laurier », fruit « semblable à celui de l'olivier »). Or généralement la nature des ressemblances n'est pas indiquée (couleur ? parfum ? forme ? consistance ?). Aussi la comparaison engendre-t-elle d'irrémédiables lacunes, d'autant plus que I'homoiotès s'applique non à une similitude totale mais à une ressemblance partielle ${ }^{158}$ et que l'éventail des végétaux méditerranéens de référence est par nécessité réduit (laurier, olivier, chêne vert, amandier, violier, lupin $)^{159}$. Troisièmement, certains descripteurs, pratiquant une sorte d'interpretatio botanique ${ }^{160}$, donnent à des espèces de mangrove le nom «olivier » ou « laurier » ${ }^{161}$. Ce faisant, ils annulent toute des cription, puisque le nom générique, tout en véhiculant sans doute des informations fondamentales (probablement que ces arbres exotiques sont sempervirents, que leur feuille est coriace et pleine, qu'ils fleurissent, donnent des fruits...), renvoie le lecteur à une réalité méditerranéenne. Cet usage élève donc l'approximation à son plus haut degré.

Plus rarement, les insuffisances que recèlent les informations antiques tiennent à la rédaction confuse de certains auteurs, inadaptée aux attentes modernes. Tantôt les formules sont sibyllines : que penser du « laurier semblable au chêne vert ${ }^{162}$ » ? Tantôt l'organisation du texte brouille les idées. Ainsi ce passage d'Arrien ${ }^{163}$, dans lequel il ardu, voire impossible, de savoir si

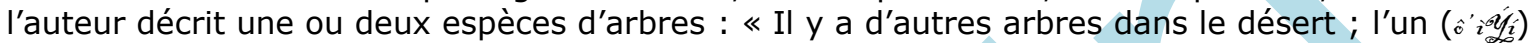
ressemble par sa feuille au laurier; celui-ci pousse dans les secteurs inondés par la mer ; à marée basse, les arbres sont laissés au sec, mais au flux, ils paraissent pousser dans la mer; les racines d'autres $\left(\hat{i} \hat{S}_{i} \ddot{a}^{\prime} \mathcal{J}^{164}\right.$ qui croissent dans des endroits creux, sont toujours recouvertes par la mer $(\ldots)^{\prime \prime}$. Le pronom ôs Siäc est équivoque : s'agit-il ici d'une deuxième espèce, que l'auteur caractérise seulement par son immersion permanente? Ou seraient-ce des individus de la première espèce, soumis à des conditions de vie un peu particulières en raison de leur emplacement. Pour prendre un autre exemple, dans un passage des Recherches sur les plantes, il est impossible de savoir

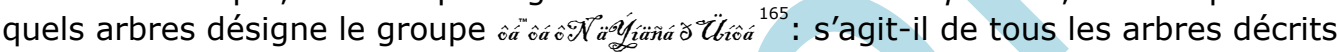
précédemment (arbres à fleur de violier, arbres à fruit semblable à celui du lupin, arbres à fruits semblable à l'amande) ou des derniers de la liste?

\section{4) Le paysage de mangrove dans l'Antiquité}

Les observateurs qui ont approché la mangrove ont décrit des arbres ; mais leur vision ne se réduit pas à des notices botaniques : leur regard embrassait un paysage nouveau. Quelques traces dans les sources laissent deviner ce qu'en fut leur perception, que le publicdes GrécoRomains cultivés a dû partager.

\section{1) Les composantes majeures : arbres et marées}

Le paysage de mangrove des Gréco-Romains se définit par l'association à parts égales de deux composantes : les arbres et la mer. En effet, dans la plupart des textes, y compris les plus succincts, apparaît ce couple indissociable : «Pythagoras (...) affirme que les Troglodytes confectionnent la pandoura à partir du (bois de) ce laurier qui pousse dans la mer »; « Le côté de I'île (de Taprobane) est baigné par une mer de couleur très verte, pleine d'arbrisseaux ${ }^{166} \gg$. La strate végétale est exclusivement arborescente, puisqu'elle est capable d'émerger. Le lexique des

158 S. Amigues, Recherches, t. 2, p. 260.

159 Voir Théophraste, $H$. P., 1, 10, 4 : en ce qui concerne la feuille, il s'agit simplement de donner une idée générale de la silhouette ; Id., 4, 4, 2 (la feuille de l'arbousier d'Orient sert de point de comparaison pour l'Avicennia, mais aussi pour le cédratier).

160 La transcription de noms vernaculaires en grec n'était pas une pratique répandue.

161 Il arrive même que le nom générique soit utilisé sans qu'existe de lien de ressemblance entre le signifiant et l'objet signifié. Voir les « oliviers qui ne ressemblent pas aux oliviers de chez nous » d'Agatharchide, Érythr., 91 (Photius, 457b).

162 Théophraste, H.P., 4, 7, 2.

163 Arrien, Anab., 6, 22, 6 .

$164 \mathrm{Ou}$ « ceux d'entre eux qui poussent dans les endroits creux ont leurs racines qui baignent en permanence dans l'eau de mer... » (trad. P. Savinel).

165 Théophraste, H. P., 4, 7, 5.

166 Respectivement Athénée, 4, 82, 184a ; Solin, 53, 21 (trad. J. André). 
textes montre bien que le Gréco-Romain n'admettait dans ce paysage que des végétaux avec

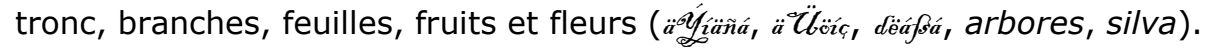

La confluence de l'élément végétal et de l'élément marin donne lieu à des représentations variées. Tantôt les deux composantes sont figurées côte à côte, les arbres sur le littoral, au voisinage immédiat de la mer : «Sur tout le littoral (du golfe Arabique) se trouvent des palmiers, des oliviers et des lauriers, non seulement dans la partie située en deçà des détroits $(\ldots)^{167} \gg$. Tantôt les deux milieux se compénètrent : « Les arbres sont laissés au sec au reflux, mais à l'arrivée du flot ils paraissent pousser dans la mer »; " Tout au long du littoral de la mer Érythrée poussent en eau profonde des arbres (...) totalement émergés à marée basse et parfois totalement submergés à marée haute ${ }^{168} \gg$. Mais le plus souvent le Gréco-Romain devait se figurer tout simplement que ces arbres poussaient dans la mer : «Mégasthène (...) rapporte que dans la mer indienne poussent des arbres »; «La mer Rouge et tout l'océan oriental sont remplis de forêts » ; « dans la mer bordière (...) croissent des plantes d'une étonnante grandeur, qui poussent en eau profonde »; « les arbres et les plantes dont on dit qu'ils croissent dans la mer Érythrée »; «(...) dans la mer (...) poussent des arbres »; « ici (i.e. après Soteiros limên) des arbres poussent dans

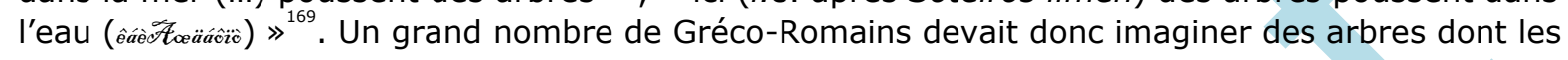
racines et une partie du tronc étaient en permanence plus ou moins immergés.

Dans la vision antique, cette association mer-arbre ne paraît évoquer ni la stabilité, ni I'harmonie. La mangrove est foncièrement instable : continuellement soumise aux variations quotidiennes du flux, elle change sans cesse : le fond vaseux apparaît ou disparaît ; le feuillage est vert quant il est immergé mais change d'aspect quand il sèche ; les amarres que l'on fixe au sommet ne remplissent plus leur office quelques heures plus tard. Surtout, dans plusieurs textes reflet de ce qui était la vision la plus courante ? - se décèle l'idée d'une opposition dynamique et violente, de sorte que I'on pourrait parler de la mangrove comme d'un paysage conflictuel. En effet, pour le Gréco-Romain, les deux éléments qui définissent la mangrove sont en lutte : la mer, élément actif, attaque les arbres, lesquels, élément passif subissent et résistent. La plus efficace arme de la mer est le sel, dont aucune partie de la plante (racine, tronc, feuille, fruit) n'est protégée, mais dont l'action ordinairement destructrice sur le vivant est sans effet sur la plante ; les racines, les feuilles, les fruits survivent et le tronc, quoique rongé, remplit ses fonctions : « Les racines de certains d'entre eux sont toujours immergées (...) sans que l'arbre ne soit abîmé par la mer »; « ceci (l'immersion) ne nuit pas à leur fruit, alors qu'il est établi que du sel reste sur les feuilles »; « cette végétation marine, tant qu'elle est en milieu humide, présente la couleur de nos algues, mais une fois hors de l'eau et exposée au soleil, en peu de temps, elle devient semblable au sel »; « rongés par le sel, semblables à des épaves abandonnées »; « tous ces arbres sont rongés en leur milieu par la mer ${ }^{170} \gg$. Pline a développé la métaphore de la lutte en y ajoutant I'action mécanique des vagues : «À la marée montante, secoués par les vagues, ils résistent, restant en place ${ }^{171} \gg$. De ce combat l'élément végétal sort encore vainqueur.

Il est fréquent que, dans les textes, l'auteur indique, plus ou moins en détails, que ces arbres sont nombreux et serrés, qu'ils constituent des peuplements denses : « les oliviers poussent en très grand nombre en ces lieux » ; (des îles) qui sont densément couvertes d'oliviers » ; « tout l'océan oriental est rempli de forêts » ; « le côté de l'île est baigné par une mer (...) pleine d'arbrisseaux »; «la partie orientale (de Tylos) est peuplée d'une telle quantité d'arbres que lorsque la mer se retire elle paraît fortifiée ${ }^{172} »$. La mangrove n'était sans doute pas perçue comme un paysage clairsemé. La palette chromatique présentait donc une dominante verte, que renforce le caractère sempervirent des arbres : «Dans la mer Érythrée verdoient des forêts »; « les (arbres) qui poussent sur terre sont grands et verts ».

\section{2) Les composantes mineure ou inexistante : hommes et animaux}

Sauf quelques exceptions, I'homme est absent du paysage que perçoivent les GrécoRomains. De rares auteurs évoquent les relations qui pouvaient unir les indigènes aux mangroves situées à proximité. La plus intéressante mention d'une présence humaine remonte à

\footnotetext{
167 Strabon 16, 4, 14.

168 Arrien, Anab., 6, 22, 6-7 ; Strabon, 16, 3, 7.

169 Antigonos, Hist. mir., 132 (Mégasthène, F. Gr. H., 133, F34), Pline, 13, 135 ; Plutarque, De facie in orbe Lunae, 25 ; Id., Mor., 911F ; Corpus aristotelicum, De mundo, 369a23 ; Strabon, 16, 4, 7. Voir également Athénée, 4, 82, 184a ; Théophraste (supra, p. )

170 Respectivement : Arrien, Anab., 6, 22, 6 ; Pline, 12, 77 ; Théophraste, H. P., 4, 7, 3 ; Pline, 12, 37 ; Théophraste, H. P., 4, 7, 5.

171 Pline, 12, 37.

172 Respectivement : Diodore de Sicile, 3, 19, 3 ; Photius, 456b (= Agatharchide, Érythr., 81) ; Pline, 13, 135 ; Solin, 53, 21 ; Théophraste, H. P., 4, 7, 7.
} 
Agatharchide $^{173}$. L'auteur signale une exploitation significative (voire une occupation) de la mangrove du golfe Arabique par des Ichtyophages. Ils confectionnent leurs abris à partir des branches d'olivier et ils y trouvent, à portée de main, leur subsistance (coquillages, crustacés, poissons). La description est accompagnée d'une réflexion sur l'adaptation des hommes à ce milieu, sur leur capacité à tirer heureusement parti de contraintes naturelles difficiles : les oliviers, qui poussent dans une région inondée régulièrement par la marée, favorisent la vie des hommes puisqu'ils les protègent du soleil tout en leur permettant de jouir de la fraîcheur marine. Ce discours est caractéristique d'une certaine ethnographie grecque.

Dans d'autres rares sources, la présence humaine est fugitivement ou implicitement signalée. Théophraste indique que des canaux, dans une mangrove du golfe Persique, étaient parcourus par des hommes. Pythagoras rapporte que les Troglodytes utilisent le bois de mangrove pour confectionner un instrument de musique ${ }^{174}$. Lorsque Androsthène affirmait qu'à Tylos le fruit d'Aeg. majus n'était pas comestible (Pline ajoute que le bétail ne le consommait pas), il y a une allusion implicite à l'utilisation des mangroves comme source de nourriture pour les hommes ou les animaux $^{175}$. Enfin, puisque les Grecs connaissaient les vertus médicinales d'Avicennia et de Rhizophora, le Moderne peut conclure que certains pénétraient plus ou moins dans la mangrove pour inciser les écorces et récolter les larmes ${ }^{176}$. Mais les témoignages antiques ne font pas explicitement allusion à cette pénétration humaine.

Aussi peut-on estimer que les Gréco-Romains concevaient généralement la mangrove comme un paysage dépourvu de présence humaine. Cette perception reflète probablement une situation réelle, car il est plausible que la présence humaine ait été rare ou inexistante dans les mangroves ou leurs alentours immédiats ${ }^{177}$. Le silence des sources serait alors le reflet d'une situation réelle. Au reste, cette hypothétique présence humaine devait être difficile à observer dès lors que I'on se contentait d'approcher la mangrove par bateau. Par ailleurs, comme la végétation était bien plus visible que les activités humaines et qu'elle attirait particulièrement l'intérêt des Méditerranéens, il n'est guère étonnant que I’homme ait été relégué au second plan.

En l'état actuel de la documentation, nulle faune ne peuple ce paysage antique. Or les mangroves abritent généralement une aquafaune riche, dans la mesure où les éléments nutritifs sont abondants, et dépendant de cette aquafaune, une avifaune souvent spectaculaire. Il est des animaux qui, d'un point de vue moderne, caractériseraient ce paysage singulier : les différentes espèces de crabe à signal, qui fourmillent à marée basse, les échassiers, les périophtalmes, poissons capables de survivre en dehors de l'eau, dotés d'yeux proéminents, grimpant sur le tronc des arbres. Or rien de tout cela ne transparaît dans les témoignages qui ont survécu. Seuls sont signalés par Agatharchide les oiseaux méléagrides (sans aucun doute des pintades) dans les îles de la mer Rouge. Mais ce ne sont pas des oiseaux de mangrove. On est d'autant plus surpris de ce silence que I'on connaît l'intérêt des compagnons d'Alexandre pour la zoologie. Ce déficit d'observation résulte peut-être de la disparition de nos sources ; mais il n'est pas exclu, une fois encore, que la prééminence de l'élément végétal n'ait relégué dans l'ombre tout ce qui appartenait au règne animal.

\section{3) La composante affective : un paysage déroutant}

Certains paysages exotiques, telles les forêts tropicales de I'Inde ou les déserts de Gédrosie, étaient plus ou moins négativement connotés dans les représentations antiques. Pour le Gréco-Romain, lecteur des récits des compagnons d'Alexandre, les éléments naturels pouvaient s'y déchaîner (ainsi les fleuves indiens en crue lors des moussons); des animaux ou des plantes dangereuses pouvaient y être rencontrés. En comparaison, la mangrove apparaît comme un paysage neutre, c'est-à-dire ni hostile ni accueillant. Il ne s'y trouve pas de créatures malfaisantes, pas d'obstacles, mais pas davantage de ressources et de secours (exception faite du mouillage). Ceci s'explique sans doute par le fait que, le plus souvent, les voyageurs approchèrent la mangrove de l'extérieur, plus qu'il ne la traversèrent ; elle fut en quelque sorte plus vue qu'expérimentée.

En revanche la dimension paradoxale parcourt profondément la perception affective du paysage de mangrove. Celui-ci étonne puissamment l'homme de l'Antiquité et bouleverse sa

173 Photius, 450b (= Agatharchide, Érythr., 43) ; Diodore de Sicile, 3, 19, 3. Les passages répétés en mer Rouge à l'époque des premiers Lagides, un souci plus marqué de l'observation ethnographique, ont peut-être permis que la présence humaine fût mieux perçue.

174 Athénée, 4, 82, 184a.

175 Théophraste, H.P., 4, 7, 7 ; Pline, 12, 38.

176 Théophraste, H. P., 4, 7, 2.

177 Ce qui est encore le cas aujourd'hui : «All but a few subsistence populations saw mangroves areas as inhospitable, unhealthy and dangerous. (...) Few community of people actually lived within the mangroves forests » (WMA, p. 11). 
perception ordinaire du monde : «Il (Agatharchide) affirme (...) qu'il se produit cette chose

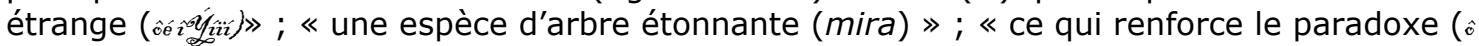
วай moyen d'oppositions fortes et significatives : "les racines de certains d'entre eux sont recouvertes par la mer (...) et cependant (ấr "iùo ils ne sont pas attaqués $(\ldots)^{\prime}$; "ceci ne nuit pas au fruit, alors qu'il est établi (cum constet) que du sel (...)"; "mais [asyndète dans le texte] dans la mer (...) poussent des arbres $(\ldots)^{179}$.

On sait ce qui rend une créature, un objet ou un paysage paradoxal: son spectacle doit contredire l'expérience courante, offrir au regard des formes incroyables, monstrueuses ou enchanteresses ; dans une certaine mesure, le paradoxon échappe aussi à la rationalité. Tout ceci est présent dans la mangrove, paysage dans lequel trois formes de paradoxal coexistent. La première naît de l'aspect étrange de la végétation, qui est source d'étonnement et définit un paradoxal d'ordre visuel : « tous ces arbres sont rongés en leur milieu par la mer et, à la façon dont ils se tiennent sur leurs racines, on dirait des poulpes »; « dès qu'ils sont exposés au soleil, en peu de temps ils prennent l'apparence du sel $\gg 180$.

Un second ordre de paradoxal se situe au niveau de la rationalité. La mangrove offre le spectacle d'une vie végétale inintelligible dans son foisonnement, parce qu'elle se développe dans un milieu, qui du point de vue gréco-romain, est hostile et stérile : «Tous (sc. les oliviers) croissent alors qu'ils sont recouverts par la mer au flux et sont constamment dans l'eau de mer au reflux » ; « les oliviers poussent véritablement en grand nombre alors que la mer baigne l'appareil racinaire » ; ces arbres parviennent même à atteindre une étonnante hauteur ( « leur taille est étonnante »; « des arbres d'une incroyable grandeur ») et parviennent au plus haut développement de la vie végétale puisqu'ils fructifient (« quant à ces arbres qui poussent dans les eaux de la marée basse, qui sont grands et portent des fruits $\gg^{181}$ ).

La mangrove est comme une sorte de renversement de l'ordre du monde, ce qui fait naître une troisième forme d'étonnement. Les arbres poussent là où ils ne devraient pas se trouver tandis que, face à la mangrove, la terre, qui devrait porter de la végétation, est vide. L'élément liquide supplante dans sa fonction le sol, devenu aussi inapte à porter de la végétation que l'est ordinairement la mer : «Sur terre, pas un arbre ne pousse (...) mais dans la mer, il y en a (..) » ; « La Gédrosie et la Troglodytique (...) sont stériles (...) et absolument dépourvues d'arbres, mais dans la mer (...) »; « Tout au long du littoral de la mer Érythrée poussent en eau profonde des arbres (...) alors que la terre dominant le rivage est sans arbres ${ }^{182} \gg$. Sans doute cette forme de paradoxal autorisa-t-elle les effets littéraires de certains auteurs, brodant sur ce baroque désordre des éléments où terre et mer se confondent : la mer recouvre des forêts (silvas operit aestus) ; la mer est peuplée d'arbres (mare ibi colore perviridi, praeterea fruticosum arboribus) ; les gouvernails des navigateurs frôlent la cime des arbres (iubas earum gubernaculis detergentibus) ${ }^{183}$. Les compagnons d'Alexandre, qui attachaient les amarres aux cimes des arbres à marée haute, avaient véritablement vécu cette étrange expérience qui brouillait les repères et rappelle le déluge que dépeint Ovide : «Entre la mer et la terre, nulle différence n'apparaissait plus (...) Celui-là navigue au-dessus de son champ de blé ou du toit de sa ferme submergée ; celui-ci prend un poisson au sommet $d^{\prime} u n$ orme ${ }^{184} \gg$.

Sous le rapport du paradoxal, la mangrove s'apparente fortement à d'autres paysages exotiques, tels ceux que les compagnons d'Alexandre avaient découverts en Inde. Toutefois, la puissance paradoxale de la mangrove dut être inférieure à celle de ces derniers, car, dans l'état actuel de la documentation, elle n'apparaît que dans un unique ouvrage paradoxographique, celui d'Antigonos, avant de disparaître de la tradition antique et de sombrer pour longtemps dans I'oubli du savoir occidental.

Pierre Schneider

178 Photius, 460a (= Agatharchide, Érythr., 108) ; Pline, 12, 37 ; Strabon, 16, 3, 7.

179 Arrien, Anab., 6, 22, 6 ; Pline, 12, 77 ; Plutarque, De facie in orbe Lunae, 25.

180 Théophraste, H. P., 4, 7, $5 ; 4,7,3$.

181 Respectivement : Photius, 460a (= Agatharchide, Érythr., 108) ; Diodore de Sicile, 3, 19, 3 ; Pline, 12, 37 ; Plutarque, De facie in orbe Lunae, 25 ; Théophraste, C. P, 2, 5, 2.

182 Théophraste, H. P., 4, 7, 1-2 ; Plutarque, De facie in orbe Lunae, 25 ; Strabon, 16, 3, 7. Cf. aussi Arrien, Anab., 6, 22, 6-7 etc. Il en reste quelque chose encore aujourd'hui : « In the Red Sea and the Gulf, despite the low diversity and relatively low total areas, the mangroves are of considerable interest and often represent the only forest habitats in the coastal areas of these countries » (WMA, p. 157).

183 Pline, 13, 141 ; Solin, 52, 49.

184 Ovide, Métam., 1, $292 ;$ 295-296. 


$$
e^{e^{2 e^{2}}}
$$




\section{Bibliographie et abréviations}

Aujac (G.), Strabon = Strabon et la science de son temps,

Amigues (S.), Recherches = Théophraste, Recherches sur les plantes, éd., trad. et comm. par S. Amigues, tome 1, Paris, 1988 ; tome 2, Paris, 1989

- , Études = Études de botanique antique, Paris, 2002

Bretzl (H.), B.F. = Botanische Forschungen des Alexanderzuges, Leipzig, 1903

Brown (T.S.), Onesicritus = Onesicritus. A Story in Hellenistic Historiography, Berkeley, 1949

Brun (J.-P.), Hodos Myshormitikè = Hodos Myshormitikè : l'équipement de la route entre Coptos et la mer Rouge aux époques ptolémaïque et romaine, dans Topoi, suppl. 3, 2002, p. 395-414

Boucharlat (R.) et Mouton (M.), Importations = Importations occidentales et influences de I'hellénisme dans la péninsule d'Oman, dans A. Invernizzi et J.-Fr. Salles (edd), Arabia antiqua. Hellenistic centers around Arabia, Rome, 1993, p. 275-289

Casson (L.), The Periplus = The Periplus Maris Erythraei, Princeton, 1989

F.Gr.H. = F. Jacoby, Die Fragmente der griechischen Historiker, Leyde, 1964-1993

Joret (Ch.), Les recherches botaniques $=$ Les recherches botaniques de l'expédition d'Alexandre (compte-rendu de l'ouvrage de H. Bretzl), dans Journal des Savants, 1904, p. 498-504 et p. 611-620

Högemann $(\mathrm{P})$, Arabien $=$ Alexander der Grossen und Arabien, Münich, 1985

Karttunen (K.), India = India and the Hellenistic World, Helsinki, 1997

Pearson (L.), Lost Histories = The Lost Histories of Alexander the Great, New York, 1960

Pédech (P.), Historiens = Historiens compagnons d'Alexandre, Paris, 1984

- , La découverte de I'Inde = La découverte de I'Inde après Alexandre, dans Cahiers du CEPOA, 6, 1995

Potts (D.T.), The Arabian Gulf = The Arabian Gulf in Antiquity, vol. 2, From Alexander the Great to the Coming of Islam, Oxford, 1990

R.E. = Pauly (A.), Wissowa (G.) et al., Realencyclopädie der Klassischen Altertumswissenschaften

Reddé (M.) et Golvin (J.-Cl.), Du Nil à la mer Rouge, dans Karthago, 21, 1986-1987, p. 5-64

Salles (J.-F.), - , Découvertes = Découvertes du golfe Arabo-Persique aux époques grecque et romaine, dans Revue des études anciennes, 94, 1992, n¹-2, p. 79-97

- , Hellénisme = Hellénisme et traditions orientales à Failaka, dans A. Invernizzi et J.-Fr. Salles (edd), Arabia antiqua. Hellenistic centers around Arabia, Rome, 1993, p. 223-232

Strasburger (Fr.), Alexanders Zug = Alexanders Zug durch die Gedrosische Wüste, dans Hermes, 80,1952, p. $456-493$

Tarn (W.W.), Ptolemy II = Ptolemy II and Arabia, dans Journal of Egyptian Archaeology, 15, 1929, p. $9-25$

Tomlinson (P.B.), Mangroves = The Botany of Mangroves, Cambridge, 1994

Weerakkody (D.P.M.), Taprobanê = Taprobanê. Ancient Sri Lanka as known to Greeks and Romans, Turhout, 1997

Wet Coastal Ecosystems, éd. V.J. Chapman, Amsterdam-Oxford..., 1997

WMA = Spalding (M.), Blasco (Fr.), Field (C.) (edd), World Mangrove Atlas, Okinawa, 1997

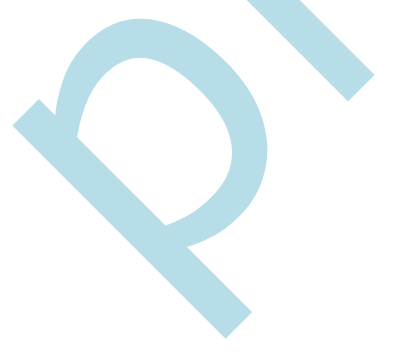

